\title{
IRRATIONALITY AND NONQUADRATICITY MEASURES FOR LOGARITHMS OF ALGEBRAIC NUMBERS
}

\author{
RAFFAELE MARCOVECCHIO and CARLO VIOLA ${ }^{\bowtie}$ \\ (Received 25 May 2011; accepted 1 February 2012) \\ Communicated by J. O. Shallit \\ Dedicated to the memory of Alf van der Poorten
}

\begin{abstract}
Let $\mathbb{K} \subset \mathbb{C}$ be a number field. We show how to compute $\mathbb{K}$-irrationality measures of a number $\xi \notin \mathbb{K}$, and $\mathbb{K}$-nonquadraticity measures of $\xi$ if $[\mathbb{K}(\xi): \mathbb{K}]>2$. By applying the saddle point method to a family of double complex integrals, we prove $\mathbb{Q}(\alpha)$-irrationality measures and $\mathbb{Q}(\alpha)$-nonquadraticity measures of $\log \alpha$ for several algebraic numbers $\alpha \in \mathbb{C}$, improving earlier results due to Amoroso and the secondnamed author.
\end{abstract}

2010 Mathematics subject classification: primary 11J82; secondary 11J17, 11J72, 30E20.

Keywords and phrases: irrationality measure, nonquadraticity measure, Weil height, saddle point method.

\section{Introduction}

In recent years, the quest for irrationality measures and nonquadraticity measures of logarithms or dilogarithms of rational numbers has been the object of deep study (see $[\mathrm{M}]$ and the bibliography therein). So far, the best quantitative results have been obtained through the asymptotic behaviour of suitable integrals of rational functions yielding linear or quadratic polynomials with rational coefficients in logarithms or dilogarithms of rational numbers, especially when one can apply to such integrals the permutation group method introduced by Rhin and the second-named author [RV1, RV2, RV3].

Typical instances of this general approach can be found in [M] (for logarithms of rational numbers) and in [RV3] (for dilogarithms of rational numbers). For example, the first-named author $[\mathrm{M}]$ recently showed that, in order to get strong results for irrationality measures and nonquadraticity measures of logarithms of positive rational

(c) 2012 Australian Mathematical Publishing Association Inc. 1446-7887/2012 \$16.00 
numbers, it is convenient to apply the Rhin-Viola permutation group method to the following family of two-dimensional complex integrals of rational functions:

$$
\begin{aligned}
& I=I(h, j, k, l, m, q ; x) \\
& =x^{\max \{0, q-l, m-h\}}(1-x)^{k+l+m+1} \\
& \quad \times \int_{s=0}^{i \infty} \int_{t=0}^{-i \infty} \frac{s^{h} t^{j}}{(1-s)^{l+k-j+1}(s-t)^{j+h-k+1}(t-x)^{k+m-h+1}} d t d s .
\end{aligned}
$$

Here $h, j, k, l, m, q$ are positive integers satisfying

$$
h+j+q=k+l+m,
$$

and such that

$$
\begin{aligned}
& l+k-j=h+q-m>0, \\
& j+h-k=m+l-q>0, \\
& k+m-h=q+j-l>0,
\end{aligned}
$$

and the parameter $x$ is a real number with $0<x<1$. In [M, Section 2], it is shown that the real and imaginary parts of the integral $I$ above have the form

$$
\begin{aligned}
\operatorname{Re}(I) & =P(x) \frac{1}{2} \log ^{2}(1 / x)-Q(x) \log (1 / x)+R(x), \\
\frac{1}{\pi} \operatorname{Im}(I) & =P(x) \log (1 / x)-Q(x),
\end{aligned}
$$

for explicitly given polynomials $P(x), Q(x), R(x) \in \mathbb{Q}[x]$ with controlled degrees and heights. By specializing to $x \in \mathbb{Q}$, the right-hand sides of the formulae (1.2) become linear and quadratic polynomials in $\log (1 / x)$ with rational coefficients $P(x), Q(x)$ and $R(x)$, and thus yield irrationality and nonquadraticity measures of $\log (1 / x)$.

The notions of irrationality and nonquadraticity measures can be extended in a natural way by defining $\mathbb{K}$-irrationality and $\mathbb{K}$-nonquadraticity measures, that is, by replacing the field $\mathbb{Q}$ with a number field $\mathbb{K} \subset \mathbb{C}$ (see Definitions 2.1 and 2.7 below). We remark that, for technical reasons, in this more general context one ought to replace the ordinary height of rational approximations by the Weil logarithmic height $h(\beta)$ of approximations $\beta \in \mathbb{K}$.

The purpose of this paper is to extend the methods of $[\mathrm{M}]$ to the Diophantine study of logarithms of algebraic numbers, by choosing the parameter $x$ in (1.1) above to be a suitable algebraic number $\alpha \in \mathbb{C}$, or any one of its algebraic conjugates (see Sections 3 and 4 below). Owing to the structure of (1.2), by choosing $x=\alpha \in \mathbb{C}$ one gets linear or quadratic polynomials in $\log (1 / \alpha)$ with coefficients $P(\alpha), Q(\alpha)$ and $R(\alpha)$ in the field $\mathbb{Q}(\alpha)$. Thus the extension of Marcovecchio's method given here naturally yields $\mathbb{Q}(\alpha)$-irrationality measures and $\mathbb{Q}(\alpha)$-nonquadraticity measures of $\log \alpha$ (the principal value of the logarithm) for several algebraic numbers $\alpha \in \mathbb{C}$. This restriction for the 
field of approximations (that is, $\mathbb{K}=\mathbb{Q}(\alpha)$ for the Diophantine study of $\log \alpha$ ) is quite natural, and is not specifically related to the integrals (1.1). The same restriction also automatically arises if one replaces the integrals (1.1) with other integrals of rational functions containing the parameter $x$, such as the integrals in [H2] or in [V].

The study of $\mathbb{Q}(\alpha)$-irrationality measures of $\log \alpha$ for suitable algebraic numbers $\alpha \in$ $\mathbb{C}$ was introduced about ten years ago by Amoroso and the second-named author [AV], and the asymptotic behaviour of the one-dimensional complex integrals involved was obtained by applying the classical saddle point method in $\mathbb{C}$. For the asymptotic study of double integrals of type (1.1) with $x \in \mathbb{C} \backslash\{0,1\}$, in this paper we require Hata's saddle point method in $\mathbb{C}^{2}$ (see [H2, Section 1]). In this way we obtain quantitative improvements on some results of [AV].

This paper is organized as follows. In Section 2 we define $\mathbb{K}$-irrationality measures and $\mathbb{K}$-nonquadraticity measures, and we prove some algebraic lemmas showing how to reduce the computation of such measures to the asymptotics of $\mathbb{K}$-approximations and of their Weil height.

In Section 3 we extend the properties of Marcovecchio's double integrals, by replacing the parameter $0<x<1$ appearing in [M] with any $x \in \mathbb{C} \backslash\{0,1\}$.

In Section 4 we specialize the complex parameter $x$ above to be an algebraic number $\alpha$, or any one of its algebraic conjugates. Using the lemmas of Section 2, we prove $\mathbb{Q}(\alpha)$-irrationality measures and $\mathbb{Q}(\alpha)$-nonquadraticity measures of $\log \alpha$ depending on the asymptotics of the double integrals, on the degree of $\alpha$ and on the leading coefficient of the minimal polynomial of $\alpha$ over $\mathbb{Z}$.

In Section 5 we discuss how to apply the saddle point method in two complex variables, in order to find the asymptotic behaviour of the double integrals of Section 3.

Finally, in Section 6 we treat numerical examples of $\mathbb{Q}(\alpha)$-irrationality measures of $\log \alpha$, improving some results in [AV], and we find $\mathbb{Q}(\alpha)$-nonquadraticity measures of $\log \alpha$ for $\alpha=\sqrt{a /(a+1)}$ with $a=6,7, \ldots, 15$.

We list here the numerical results obtained in Section 6 for the least $\mathbb{Q}(\alpha)$ irrationality measure $\mu=\mu_{\mathbb{Q}(\alpha)}(\log \alpha)$ of $\log \alpha$, for the algebraic numbers $\alpha$ considered, together with the corresponding results proved in the paper [AV]. For convenience we write $\alpha_{0}$ for the root of the equation $5 \alpha^{3}-5 \alpha^{2}+1=0$ for which $\operatorname{Im}(\alpha)>0$.

$$
\begin{array}{lll}
\alpha=1 / \sqrt{2}, & \mu \leq 11.55509194 \ldots & ([\mathrm{AV}]: 12.4288), \\
\alpha=\sqrt{2 / 3}, & \mu \leq 10.80627515 \ldots & ([\mathrm{AV}]: 12.1383), \\
\alpha=\alpha_{0}, & \mu \leq 6.93729219 \ldots & ([\mathrm{AV}]: 7.105), \\
\alpha=e^{i \pi / 6}, & \mu \leq 36.17593210 \ldots & ([\mathrm{AV}]: 46.9075) .
\end{array}
$$

The choice of the algebraic numbers $\alpha$ in (1.3) is motivated both by comparison with the corresponding numerical results previously obtained in [AV], and by the applications of the $\mathbb{C}^{2}$-saddle point method that can be made for $\alpha$ and for its algebraic conjugates. 
Since $\log \sqrt{2}=\frac{1}{2} \log 2$, the first numerical result in (1.3) can be written in the form

$$
\mu_{\mathbb{Q}(\sqrt{2})}(\log 2) \leq 11.55509194 \ldots,
$$

and, owing to the normalization used for the Weil logarithmic height, it is equivalent to the following bound:

$$
|\log 2-U|>\mathrm{H}(U)^{-5.77754597 \ldots}
$$

for any $U \in \mathbb{Q}(\sqrt{2})$ with sufficiently large (ordinary) height $\mathrm{H}(U)$ (that is, $\mathrm{H}(U)$ is the maximum of the absolute values of the coefficients of the minimal polynomial of $U$ over $\mathbb{Z}$ ). The bound (1.4) should be compared with the best known nonquadraticity measure of $\log 2$ proved in [M], namely

$$
|\log 2-U|>\mathrm{H}(U)^{-15.65142024 \ldots,}
$$

which holds for any quadratic number $U$ with sufficiently large $\mathrm{H}(U)$.

We also remark that the last bound listed in (1.3) can be written as

$$
\mu_{\mathbb{Q}(i, \sqrt{3})}(i \pi / 6)=\mu_{\mathbb{Q}(\sqrt{3})}(\pi) \leq 36.17593210 \ldots,
$$

because $e^{i \pi / 6}=\frac{1}{2}(\sqrt{3}+i)$, and therefore yields

$$
\left|\pi-\frac{a+b \sqrt{3}}{c+d \sqrt{3}}\right|>C \cdot \max \{|a|,|b|,|c|,|d|\}^{-36.17593210 \ldots}
$$

for all $a, b, c, d \in \mathbb{Z}$ with $(c, d) \neq(0,0)$, where $C$ is a constant. In particular, for $d=0$, we get the following linear independence measure of $1, \sqrt{3}, \pi$ over $\mathbb{Q}$ :

$$
|a+b \sqrt{3}+c \pi|>C \cdot|c| \cdot \max \{|a|,|b|,|c|\}^{-36.17593210 \ldots}
$$

for all $a, b, c \in \mathbb{Z}, c \neq 0$.

\section{Algebraic lemmas}

We first recall some well-known definitions and properties concerning the Weil height of algebraic numbers. Let $\mathbb{K} \subset \mathbb{C}$ be a number field, and let $\mathcal{M}_{\mathbb{K}}$ be the set of places of $\mathbb{K}$. For any $v \in \mathcal{M}_{\mathbb{K}}$, we denote by $|\cdot|_{v}$ the normalized absolute value associated with $v$, and we set $\eta_{v}=\left[\mathbb{K}_{v}: \mathbb{Q}_{v}\right]$. If $\sigma$ is an embedding of $\mathbb{K}$ in $\mathbb{C}$ and $v=v_{\sigma}$ is the associated place, then $|\beta|_{v}=|\sigma(\beta)|$ for $\beta \in \mathbb{K}$, and $\eta_{v}=1$ if $\sigma$ is real and $\eta_{v}=2$ otherwise. On the other hand, if $v \mid p$, where $p$ is a prime number, the absolute value $|\cdot|_{v}$ is normalized by taking $|p|_{v}=p^{-1}$.

The Weil absolute logarithmic height $\mathrm{h}(\beta)$ of $\beta$ is defined to be

$$
\mathrm{h}(\beta)=\frac{1}{[\mathbb{K}: \mathbb{Q}]} \sum_{v \in \mathcal{M}_{\mathbb{K}}} \eta_{v} \log ^{+}|\beta|_{v}
$$


for all $\beta \in \mathbb{K}$, where $\log ^{+} x=\log \max \{x, 1\}$ for any $x \geq 0$. It is well known that $\mathrm{h}(\beta)$ depends only on the algebraic number $\beta$, and is independent of the number field $\mathbb{K}$ containing $\beta$. Also, for any algebraic numbers $\beta_{1}$ and $\beta_{2}$,

$$
\mathrm{h}\left(\beta_{1}+\beta_{2}\right) \leq \mathrm{h}\left(\beta_{1}\right)+\mathrm{h}\left(\beta_{2}\right)+\log 2,
$$

since $\max \{x+y, 1\} \leq 2 \max \{x, 1\} \max \{y, 1\}$ for all $x \geq 0$ and $y \geq 0$.

We also recall that for any $\beta \in \mathbb{K}^{\times}$we have the product formula

$$
\sum_{v \in \mathcal{M}_{\mathbb{K}}} \eta_{v} \log |\beta|_{v}=0
$$

and the Liouville inequality

$$
\log |\beta| \geq-\delta \mathrm{h}(\beta)
$$

where

$$
\delta=\frac{[\mathbb{K}: \mathbb{Q}]}{\left[\mathbb{K}_{\infty}: \mathbb{R}\right]}= \begin{cases}{[\mathbb{K}: \mathbb{Q}]} & \text { if } \mathbb{K} \subset \mathbb{R} \\ \frac{1}{2}[\mathbb{K}: \mathbb{Q}] & \text { otherwise }\end{cases}
$$

Here $\mathbb{K}_{\infty}$ denotes the completion of the field $\mathbb{K}$ with respect to the Euclidean absolute value, that is, $\mathbb{K}_{\infty}=\mathbb{R}$ if $\mathbb{K} \subset \mathbb{R}$ and $\mathbb{K}_{\infty}=\mathbb{C}$ otherwise.

Definition 2.1. Let $\mathbb{K} \subset \mathbb{C}$ be a number field, let $\xi \in \mathbb{K}_{\infty} \backslash \mathbb{K}$, and let $\mu$ be a positive real number. We say that $\mu$ is a $\mathbb{K}$-irrationality measure of $\xi$ if for any $\varepsilon>0$ there exists a constant $h_{0}=h_{0}(\varepsilon)>0$ such that

$$
\log |\xi-\beta|>-(1+\varepsilon) \mu \mathrm{h}(\beta)
$$

for all $\beta \in \mathbb{K}$ with $\mathrm{h}(\beta)>h_{0}$. The least $\mathbb{K}$-irrationality measure of $\xi$ is denoted by $\mu_{\mathbb{K}}(\xi)$.

By the Dirichlet box principle,

$$
\mu_{\mathbb{K}}(\xi) \geq 2 \frac{[\mathbb{K}: \mathbb{Q}]}{\left[\mathbb{K}_{\infty}: \mathbb{R}\right]}=2 \delta
$$

(see [S, pp. 253 and 255]).

For the computation of $\mathbb{K}$-irrationality measures we shall use the following lemma, which yields the conclusion of [AV, Lemma 2.4] under a weaker assumption.

Lemma 2.2. Let $\mathbb{K} \subset \mathbb{C}$ be a number field, and let $\delta$ be defined by (2.5). Let $\xi \in \mathbb{C}$, and let $\left(\vartheta_{n}\right)_{n \in \mathbb{N}}$ be a sequence of elements of $\mathbb{K}$ such that $\vartheta_{n} \neq \xi$,

$$
\limsup _{n \rightarrow \infty} \frac{1}{n} \log \left|\xi-\vartheta_{n}\right| \leq-\varrho \quad \text { and } \quad \limsup _{n \rightarrow \infty} \frac{1}{n} \mathrm{~h}\left(\vartheta_{n}\right) \leq c
$$

for positive real numbers $\varrho$ and c. If

$$
\lambda:=\frac{1}{\delta}-\frac{c}{\varrho}>0
$$

then $\xi \notin \mathbb{K}$ and $\mu_{\mathbb{K}}(\xi) \leq \lambda^{-1}$. 
Proof. If we had $\xi \in \mathbb{K}$, then we would have $\xi-\vartheta_{n} \in \mathbb{K}^{\times}$. By the Liouville inequality (2.4) and (2.2),

$$
-\log \left|\xi-\vartheta_{n}\right| \leq \delta \mathrm{h}\left(\xi-\vartheta_{n}\right) \leq \delta\left(\mathrm{h}(\xi)+\mathrm{h}\left(\vartheta_{n}\right)+\log 2\right) .
$$

Dividing by $n$ and making $n \rightarrow \infty$, we get $\varrho \leq \delta c$ by (2.6), thus contradicting (2.7). Therefore $\xi \notin \mathbb{K}$.

For any $\varepsilon>0$ let $\omega=\omega(\varepsilon)>0$ satisfy

$$
0<\frac{\delta(c+\omega)}{\varrho-\delta \omega}<1<\frac{\varrho-\delta \omega}{\varrho-\delta c-2 \delta \omega}<\left(1+\frac{\varepsilon}{2}\right) \frac{\varrho}{\varrho-\delta c}=\left(1+\frac{\varepsilon}{2}\right) \frac{1}{\lambda \delta},
$$

and let $n_{0}=n_{0}(\varepsilon)>0$ be such that for any $n>n_{0}$,

$$
\log \left|\xi-\vartheta_{n}\right|<-(\varrho-\delta \omega) n \quad \text { and } \quad \mathrm{h}\left(\vartheta_{n}\right)<(c+\omega) n .
$$

Define $h_{0}$ to be

$$
\max \left\{\frac{n_{0}(\varrho-\delta c-2 \delta \omega)-(\delta+1) \log 2}{\delta}, \frac{2 \lambda}{\varepsilon}(\delta(c+\omega)+(\delta+1) \log 2) \frac{\varrho-\delta \omega}{\varrho-\delta c-2 \delta \omega}\right\} .
$$

Let $\beta \in \mathbb{K}$ with $\mathrm{h}(\beta)>h_{0}$, and let

$$
M=\left[\frac{\delta \mathrm{h}(\beta)+(\delta+1) \log 2}{\varrho-\delta c-2 \delta \omega}\right]+1,
$$

whence

$$
M>\frac{\delta h_{0}+(\delta+1) \log 2}{\varrho-\delta c-2 \delta \omega} \geq n_{0}
$$

For any $n \geq M$ such that $\vartheta_{n} \neq \beta$, we have, by (2.4), (2.2) and (2.8),

$$
\begin{aligned}
-\log \left|\vartheta_{n}-\beta\right| & \leq \delta \mathrm{h}\left(\vartheta_{n}-\beta\right) \\
& \leq \delta\left(\mathrm{h}\left(\vartheta_{n}\right)+\mathrm{h}(\beta)+\log 2\right) \\
& <\delta(c+\omega) n+\delta(\mathrm{h}(\beta)+\log 2) .
\end{aligned}
$$

Therefore, by (2.8),

$$
\begin{aligned}
\log \left|\xi-\vartheta_{n}\right|-\log \left|\vartheta_{n}-\beta\right| & <-(\varrho-\delta \omega) n+\delta(c+\omega) n+\delta(\mathrm{h}(\beta)+\log 2) \\
& \leq-(\varrho-\delta c-2 \delta \omega) M+\delta \mathrm{h}(\beta)+\delta \log 2 \\
& <-\log 2
\end{aligned}
$$

whence $\left|\xi-\vartheta_{n}\right|<\frac{1}{2}\left|\vartheta_{n}-\beta\right|$. By the triangle inequality we get $|\xi-\beta|>\frac{1}{2}\left|\vartheta_{n}-\beta\right|$, and by (2.9) this yields

$$
\begin{aligned}
-\log |\xi-\beta| & <\log 2-\log \left|\vartheta_{n}-\beta\right| \\
& <\delta(c+\omega) n+\delta \mathrm{h}(\beta)+(\delta+1) \log 2 .
\end{aligned}
$$


We distinguish two cases.

First case: $\vartheta_{M} \neq \beta$. We may take $n=M$ in (2.10). Thus

$$
\begin{aligned}
-\log |\xi-\beta| & <\delta(c+\omega) M+\delta \mathrm{h}(\beta)+(\delta+1) \log 2 \\
& \leq \delta(c+\omega)\left(\frac{\delta \mathrm{h}(\beta)+(\delta+1) \log 2}{\varrho-\delta c-2 \delta \omega}+1\right)+\delta \mathrm{h}(\beta)+(\delta+1) \log 2 \\
& =(\delta \mathrm{h}(\beta)+(\delta+1) \log 2)\left(\frac{\delta c+\delta \omega}{\varrho-\delta c-2 \delta \omega}+1\right)+\delta(c+\omega) \\
& <\delta \mathrm{h}(\beta) \frac{\varrho-\delta \omega}{\varrho-\delta c-2 \delta \omega}+(\delta(c+\omega)+(\delta+1) \log 2) \frac{\varrho-\delta \omega}{\varrho-\delta c-2 \delta \omega} \\
& <\left(1+\frac{\varepsilon}{2}\right) \frac{\mathrm{h}(\beta)}{\lambda}+\frac{\varepsilon h_{0}}{2 \lambda} \\
& <\frac{1+\varepsilon}{\lambda} \mathrm{h}(\beta) .
\end{aligned}
$$

Second case: $\vartheta_{M}=\beta$. By (2.6) we have $\lim _{n \rightarrow \infty} \vartheta_{n}=\xi \neq \beta$ since $\beta \in \mathbb{K}$ and $\xi \notin \mathbb{K}$. Thus $\vartheta_{n} \neq \beta$ for all sufficiently large $n$. Let $N$ be the least integer such that $N>M$ and $\vartheta_{N} \neq \beta$. Then, by (2.8) with $n=N-1$,

$$
\log |\xi-\beta|=\log \left|\xi-\vartheta_{N-1}\right|<-(\varrho-\delta \omega)(N-1),
$$

that is,

$$
N<-\frac{\log |\xi-\beta|}{\varrho-\delta \omega}+1
$$

Taking $n=N$ in (2.10) we obtain

$$
\begin{aligned}
-\log |\xi-\beta| & <\delta(c+\omega) N+\delta \mathrm{h}(\beta)+(\delta+1) \log 2 \\
& <-\frac{\delta(c+\omega) \log |\xi-\beta|}{\varrho-\delta \omega}+\delta(c+\omega)+\delta \mathrm{h}(\beta)+(\delta+1) \log 2
\end{aligned}
$$

whence

$$
-\left(1-\frac{\delta(c+\omega)}{\varrho-\delta \omega}\right) \log |\xi-\beta|<\delta(c+\omega)+\delta \mathrm{h}(\beta)+(\delta+1) \log 2
$$

Thus

$$
-\log |\xi-\beta|<\frac{\varrho-\delta \omega}{\varrho-\delta c-2 \delta \omega}(\delta(c+\omega)+\delta \mathrm{h}(\beta)+(\delta+1) \log 2) .
$$

This yields, as in the first case,

$$
-\log |\xi-\beta|<\left(1+\frac{\varepsilon}{2}\right) \frac{\mathrm{h}(\beta)}{\lambda}+\frac{\varepsilon h_{0}}{2 \lambda}<\frac{1+\varepsilon}{\lambda} \mathrm{h}(\beta) .
$$

This concludes the proof.

We notice that, as a consequence of Lemma 2.2 above, we easily get a proof of [H1, Remark 2.1, p. 339]. 
Corollary 2.3 (Hata [H1]). Let $\xi \in \mathbb{R} \backslash \mathbb{Q}$, and let $\left(p_{n}\right)_{n \in \mathbb{N}}$ and $\left(q_{n}\right)_{n \in \mathbb{N}}$ be sequences of integers such that

$$
\lim _{n \rightarrow \infty} \frac{1}{n} \log \left|q_{n}\right|=\sigma \quad \text { and } \quad \limsup _{n \rightarrow \infty} \frac{1}{n} \log \left|q_{n} \xi-p_{n}\right| \leq-\tau
$$

for positive real numbers $\sigma$ and $\tau$. Then

$$
\mu(\xi)=\mu_{\mathbb{Q}}(\xi) \leq 1+\frac{\sigma}{\tau} .
$$

Proof. Let $\vartheta_{n}=p_{n} / q_{n}$ and $\mathbb{K}=\mathbb{Q}$. We observe that

$$
\begin{aligned}
\limsup _{n \rightarrow \infty} \frac{1}{n} \log \left|\xi-\vartheta_{n}\right| & =\limsup _{n \rightarrow \infty} \frac{1}{n}\left(\log \left|q_{n} \xi-p_{n}\right|-\log \left|q_{n}\right|\right) \\
& \leq \limsup _{n \rightarrow \infty} \frac{1}{n} \log \left|q_{n} \xi-p_{n}\right|-\liminf _{n \rightarrow \infty} \frac{1}{n} \log \left|q_{n}\right| \\
& \leq-(\sigma+\tau) .
\end{aligned}
$$

Moreover, $\mathrm{h}\left(\vartheta_{n}\right) \leq \log \max \left\{\left|p_{n}\right|,\left|q_{n}\right|\right\}$, with equality if and only if $\left(p_{n}, q_{n}\right)=1$, and

$$
p_{n}=q_{n} \xi\left(1-\frac{q_{n} \xi-p_{n}}{q_{n} \xi}\right),
$$

whence

$$
\lim _{n \rightarrow \infty} \frac{1}{n} \log \left|p_{n}\right|=\lim _{n \rightarrow \infty} \frac{1}{n} \log \left|q_{n}\right|=\sigma .
$$

From Lemma 2.2 with $\lambda=1-\sigma /(\sigma+\tau)=\tau /(\sigma+\tau)$

$$
\mu(\xi) \leq \lambda^{-1}=1+\frac{\sigma}{\tau}
$$

as required.

In order to suitably define a $\mathbb{K}$-nonquadraticity measure of a number, and to prove a statement allowing computation of $\mathbb{K}$-nonquadraticity measures, it is convenient to first introduce $\mathbb{K}$-linear independence measures. Let $\mathbb{K} \subset \mathbb{C}$ be a number field, and let $\left(\vartheta_{0}: \vartheta_{1}: \vartheta_{2}\right)$ be a point in the projective plane $\mathbb{P}^{2}(\mathbb{K})$, with homogeneous coordinates $\vartheta_{0}, \vartheta_{1}, \vartheta_{2} \in \mathbb{K}$ not all zero. By analogy with (2.1), we define the Weil absolute logarithmic height of $\left(\vartheta_{0}: \vartheta_{1}: \vartheta_{2}\right)$ by

$$
\mathrm{h}\left(\left(\vartheta_{0}: \vartheta_{1}: \vartheta_{2}\right)\right)=\frac{1}{[\mathbb{K}: \mathbb{Q}]} \sum_{v \in \mathcal{M}_{\mathbb{K}}} \eta_{v} \log \max \left\{\left|\vartheta_{0}\right|_{v},\left|\vartheta_{1}\right|_{v},\left|\vartheta_{2}\right|_{v}\right\} .
$$

It is easy to see that $h\left(\left(\vartheta_{0}: \vartheta_{1}: \vartheta_{2}\right)\right)$ is independent of the number field $\mathbb{K}$ and, by the product formula (2.3), it is also independent of the choice of the homogeneous coordinates $\vartheta_{0}, \vartheta_{1}, \vartheta_{2}$. Moreover, for any $\left(1: \sigma_{1}: \sigma_{2}\right),\left(1: \vartheta_{1}: \vartheta_{2}\right),\left(1: \tau_{1}: \tau_{2}\right) \in$ $\mathbb{P}^{2}(\mathbb{K})$ it is easily seen that

$$
\mathrm{h}\left(\sigma_{1} \vartheta_{2}+\tau_{1} \sigma_{2}+\vartheta_{1} \tau_{2}\right) \leq \mathrm{h}\left(\left(1: \sigma_{1}: \sigma_{2}\right)\right)+\mathrm{h}\left(\left(1: \vartheta_{1}: \vartheta_{2}\right)\right)+\mathrm{h}\left(\left(1: \tau_{1}: \tau_{2}\right)\right)+\log 3,
$$


similarly to (2.2), since

$$
\max \left\{x_{1} y_{2}+z_{1} x_{2}+y_{1} z_{2}, 1\right\} \leq 3 \max \left\{1, x_{1}, x_{2}\right\} \max \left\{1, y_{1}, y_{2}\right\} \max \left\{1, z_{1}, z_{2}\right\}
$$

for $x_{1}, x_{2}, y_{1}, y_{2}, z_{1}, z_{2} \geq 0$. In particular, for $\vartheta_{1}=\vartheta_{2}=1$,

$$
\mathrm{h}\left(\sigma_{1}+\tau_{1} \sigma_{2}+\tau_{2}\right) \leq \mathrm{h}\left(\left(1: \sigma_{1}: \sigma_{2}\right)\right)+\mathrm{h}\left(\left(1: \tau_{1}: \tau_{2}\right)\right)+\log 3
$$

because $\mathrm{h}\left(\left(1: \vartheta_{1}: \vartheta_{2}\right)\right)=\mathrm{h}((1: 1: 1))=0$.

Definition 2.4. Let $\xi_{0}, \xi_{1}, \xi_{2} \in \mathbb{K}_{\infty}$ be linearly independent over $\mathbb{K}$. We say that $\mu>0$ is a $\mathbb{K}$-linear independence measure of $\xi_{0}, \xi_{1}, \xi_{2}$ over $\mathbb{K}$ if for any $\varepsilon>0$ there exists a constant $h_{0}=h_{0}(\varepsilon)>0$ such that

$$
\log \frac{\left|p \xi_{0}+q \xi_{1}+r \xi_{2}\right|}{\max \{|p|,|q|,|r|\}}>-(1+\varepsilon) \mu \mathrm{h}((p: q: r))
$$

for all $(p: q: r) \in \mathbb{P}^{2}(\mathbb{K})$ with $\mathrm{h}((p: q: r))>h_{0}$. The least $\mathbb{K}$-linear independence measure of $\xi_{0}, \xi_{1}, \xi_{2}$ is denoted by $\mu_{\mathbb{K}}\left(\left(\xi_{0}: \xi_{1}: \xi_{2}\right)\right)$.

REMARK 2.5. Plainly $\mu_{\mathbb{K}}\left(\left(\xi_{0}: \xi_{1}: \xi_{2}\right)\right)$ is invariant if we replace $\xi_{0}, \xi_{1}$ and $\xi_{2}$ by $t \xi_{0}, t \xi_{1}$ and $t \xi_{2}$, for any $t \neq 0$. Also, in inequality (2.13) one can replace $\max \{|p|,|q|,|r|\}$ by $\max \{|q|,|r|\}$ without changing the definition of $\mathbb{K}$-linear independence measure. For if $|p|>\max \{|q|,|r|\}$ and $\left|p \xi_{0}+q \xi_{1}+r \xi_{2}\right| /|p|<\frac{1}{2}\left|\xi_{0}\right|$, which is clearly not restrictive, then

$$
\begin{aligned}
\left|p \xi_{0}\right| & \leq\left|p \xi_{0}+q \xi_{1}+r \xi_{2}\right|+\left(\left|\xi_{1}\right|+\left|\xi_{2}\right|\right) \max \{|q|,|r|\} \\
& \leq \frac{1}{2}\left|p \xi_{0}\right|+\left(\left|\xi_{1}\right|+\left|\xi_{2}\right|\right) \max \{|q|,|r|\}
\end{aligned}
$$

whence

$$
\max \{|q|,|r|\}<|p| \leq 2 \frac{\left|\xi_{1}\right|+\left|\xi_{2}\right|}{\left|\xi_{0}\right|} \max \{|q|,|r|\} .
$$

Lemma 2.6. Let $\mathbb{K} \subset \mathbb{C}$ be a number field, and let $\delta$ be defined by (2.5). Let $\xi_{1}, \xi_{2} \in \mathbb{C}$, and let $\left(\varphi_{n}\right)_{n \in \mathbb{N}}$ and $\left(\psi_{n}\right)_{n \in \mathbb{N}}$ be two sequences of elements of $\mathbb{K}$ such that $\xi_{1}-\varphi_{n}$ and $\xi_{2}-\psi_{n}$ are linearly independent over $\mathbb{K}$, and

$$
\begin{gathered}
\max \left\{\limsup _{n \rightarrow \infty} \frac{1}{n} \log \left|\xi_{1}-\varphi_{n}\right|, \limsup _{n \rightarrow \infty} \frac{1}{n} \log \left|\xi_{2}-\psi_{n}\right|\right\} \leq-\varrho \\
\limsup _{n \rightarrow \infty} \frac{1}{n} \mathrm{~h}\left(\left(1: \varphi_{n}: \psi_{n}\right)\right) \leq c
\end{gathered}
$$

for positive real numbers $\varrho$ and $c$. If

$$
\lambda:=\frac{1}{\delta}-\frac{c}{\varrho}>0
$$

then $1, \xi_{1}, \xi_{2}$ are linearly independent over $\mathbb{K}$, and

$$
\mu_{\mathbb{K}}\left(\left(1: \xi_{1}: \xi_{2}\right)\right) \leq \lambda^{-1} .
$$


Proof. The proof follows the same scheme as Lemma 2.2. If we had $p+q \xi_{1}+r \xi_{2}=0$ for suitable $p, q, r \in \mathbb{K}$ with $(q, r) \neq(0,0)$, up to a permutation we could assume that $r=1$, so that

$$
p+q \xi_{1}+\xi_{2}=0
$$

Since $\xi_{1}-\varphi_{n}$ and $\xi_{2}-\psi_{n}$ are linearly independent over $\mathbb{K}$,

$$
q\left(\xi_{1}-\varphi_{n}\right)+\xi_{2}-\psi_{n} \neq 0
$$

whence, by (2.16),

$$
p+q \varphi_{n}+\psi_{n}=q\left(\varphi_{n}-\xi_{1}\right)+\psi_{n}-\xi_{2} \neq 0
$$

Since

$$
\left|q\left(\varphi_{n}-\xi_{1}\right)+\psi_{n}-\xi_{2}\right| \leq(|q|+1) \max \left\{\left|\varphi_{n}-\xi_{1}\right|,\left|\psi_{n}-\xi_{2}\right|\right\},
$$

using the Liouville inequality (2.4) and (2.12) we obtain

$$
\begin{aligned}
& -\log (|q|+1)-\log \max \left\{\left|\varphi_{n}-\xi_{1}\right|,\left|\psi_{n}-\xi_{2}\right|\right\} \leq-\log \left|p+q \varphi_{n}+\psi_{n}\right| \\
& \leq \delta \mathrm{h}\left(p+q \varphi_{n}+\psi_{n}\right) \leq \delta\left(\mathrm{h}\left(\left(1: \varphi_{n}: \psi_{n}\right)\right)+\mathrm{h}((1: p: q))+\log 3\right),
\end{aligned}
$$

whence, dividing by $n$ and making $n \rightarrow \infty$, by (2.14) we get $\varrho \leq \delta c$, contradicting (2.15). Therefore $1, \xi_{1}, \xi_{2}$ are linearly independent over $\mathbb{K}$.

For any $\varepsilon>0$ let $\omega=\omega(\varepsilon)>0$ be as in the proof of Lemma 2.2, let $n_{0}=n_{0}(\varepsilon)>0$ be such that

$$
\begin{gathered}
\log \max \left\{\left|\xi_{1}-\varphi_{n}\right|,\left|\xi_{2}-\psi_{n}\right|\right\}<-(\varrho-\delta \omega) n, \\
\mathrm{~h}\left(\left(1: \varphi_{n}: \psi_{n}\right)\right)<(c+\omega) n
\end{gathered}
$$

for any $n>n_{0}$, and let $h_{0}$ be

$$
\max \left\{\frac{n_{0}(\varrho-\delta c-2 \delta \omega)-(\delta+1) \log 3}{\delta}, \frac{2 \lambda}{\varepsilon}(\delta(c+\omega)+(\delta+2) \log 3) \frac{\varrho-\delta \omega}{\varrho-\delta c-2 \delta \omega}\right\} .
$$

Let $(p: q: r) \in \mathbb{P}^{2}(\mathbb{K})$ with $\mathrm{h}((p: q: r))>h_{0}$. By Remark 2.5, we must prove that

$$
\log \frac{\left|p+q \xi_{1}+r \xi_{2}\right|}{\max \{|q|,|r|\}}>-(1+\varepsilon) \mu \mathrm{h}((p: q: r)) \text {. }
$$

Since both sides of this inequality depend only on $(p: q: r)$, up to a permutation we may assume that $|q| \leq 1=r$. Define

$$
M=\left[\frac{\delta \mathrm{h}((1: p: q))+(\delta+1) \log 3}{\varrho-\delta c-2 \delta \omega}\right]+1>n_{0} .
$$

For any $n \geq M$ such that $p+q \varphi_{n}+\psi_{n} \neq 0$, by (2.12) and (2.17),

$$
\begin{aligned}
-\log \left|p+q \varphi_{n}+\psi_{n}\right| & \leq \delta \mathrm{h}\left(p+q \varphi_{n}+\psi_{n}\right) \\
& \leq \delta\left(\mathrm{h}\left(\left(1: \varphi_{n}: \psi_{n}\right)\right)+\mathrm{h}((1: p: q))+\log 3\right) \\
& <\delta(c+\omega) n+\delta(\mathrm{h}((1: p: q))+\log 3),
\end{aligned}
$$


similarly to (2.9). Therefore, by (2.17),

$$
\begin{aligned}
\log \mid & q\left(\xi_{1}-\varphi_{n}\right)+\xi_{2}-\psi_{n}|-\log | p+q \varphi_{n}+\psi_{n} \mid \\
& \leq \log \left(2 \max \left\{\left|\xi_{1}-\varphi_{n}\right|,\left|\xi_{2}-\psi_{n}\right|\right\}\right)+\delta(c+\omega) n+\delta(\mathrm{h}((1: p: q))+\log 3) \\
& \leq-(\varrho-\delta \omega) n+\log 2+\delta(c+\omega) n+\delta \mathrm{h}((1: p: q))+\delta \log 3 \\
& \leq-(\varrho-\delta c-2 \delta \omega) M+\delta \mathrm{h}((1: p: q))+\delta \log 3+\log 2 \\
& <-\log (3 / 2),
\end{aligned}
$$

whence

$$
\left|q\left(\xi_{1}-\varphi_{n}\right)+\xi_{2}-\psi_{n}\right|<\frac{2}{3}\left|p+q \varphi_{n}+\psi_{n}\right| .
$$

Thus, by the triangle inequality,

$$
\left|p+q \xi_{1}+\xi_{2}\right|>\frac{1}{3}\left|p+q \varphi_{n}+\psi_{n}\right|
$$

so that

By (2.18),

$$
-\log \left|p+q \xi_{1}+\xi_{2}\right|<\log 3-\log \left|p+q \varphi_{n}+\psi_{n}\right|
$$

$$
-\log \left|p+q \xi_{1}+\xi_{2}\right|<\delta(c+\omega) n+\delta \mathrm{h}((1: p: q))+(\delta+1) \log 3 .
$$

As in Lemma 2.2 we distinguish two cases.

First case: $p+q \varphi_{M}+\psi_{M} \neq 0$. We take $n=M$ in (2.19) and, similarly to Lemma 2.2, we obtain

$$
\begin{aligned}
-\log \left|p+q \xi_{1}+\xi_{2}\right| & <\delta(c+\omega) M+\delta \mathrm{h}((1: p: q))+(\delta+1) \log 3 \\
& <\left(1+\frac{\varepsilon}{2}\right) \frac{\mathrm{h}((1: p: q))}{\lambda}+\frac{\varepsilon h_{0}}{2 \lambda} \\
& <\frac{1+\varepsilon}{\lambda} \mathrm{h}((1: p: q)) .
\end{aligned}
$$

Second case: $p+q \varphi_{M}+\psi_{M}=0$. Since $\lim _{n \rightarrow \infty} \varphi_{n}=\xi_{1}$ and $\lim _{n \rightarrow \infty} \psi_{n}=\xi_{2}$, we may take the least integer $N$ such that $N>M$ and $p+q \varphi_{N}+\psi_{N} \neq 0$. Then, by (2.17) with $n=N-1$,

$$
\begin{aligned}
\log \left|p+q \xi_{1}+\xi_{2}\right| & =\log \left|q\left(\xi_{1}-\varphi_{N-1}\right)+\xi_{2}-\psi_{N-1}\right| \\
& <-(\varrho-\delta \omega)(N-1)+\log 2,
\end{aligned}
$$

whence

$$
N<\frac{\log 2-\log \left|p+q \xi_{1}+\xi_{2}\right|}{\varrho-\delta \omega}+1 .
$$

Taking $n=N$ in (2.19), we easily obtain

$$
\begin{aligned}
-\log \left|p+q \xi_{1}+\xi_{2}\right| & <\left(1+\frac{\varepsilon}{2}\right) \frac{\mathrm{h}((1: p: q))}{\lambda}+\frac{\varepsilon h_{0}}{2 \lambda} \\
& <\frac{1+\varepsilon}{\lambda} \mathrm{h}((1: p: q)),
\end{aligned}
$$

as required. 
Definition 2.7. Let $\mathbb{K} \subset \mathbb{C}$ be a number field, and let $\xi \in \mathbb{C}$ be nonquadratic over $\mathbb{K}$, that is, such that $[\mathbb{K}(\xi): \mathbb{K}]>2$. A real number $\mu>0$ is said to be a $\mathbb{K}$-nonquadraticity measure of $\xi$ if for any $\varepsilon>0$ there exists a constant $h_{0}=h_{0}(\varepsilon)>0$ such that

$$
\log |\xi-\beta|>-(2+\varepsilon) \mu \mathrm{h}(\beta)
$$

for all $\beta$ that are quadratic over $\mathbb{K}$, that is, satisfy $[\mathbb{K}(\beta): \mathbb{K}] \leq 2$, with $\mathrm{h}(\beta)>h_{0}$. The least $\mathbb{K}$-nonquadraticity measure of $\xi$ is denoted by $\mu_{2, \mathbb{K}}(\xi)$.

Corollary 2.8. Let $\mathbb{K} \subset \mathbb{C}$ be a number field, and let $\delta$ be defined by (2.5). Let $\xi \in \mathbb{K}_{\infty}$, and let $\left(\varphi_{n}\right)_{n \in \mathbb{N}}$ and $\left(\psi_{n}\right)_{n \in \mathbb{N}}$ be two sequences of elements of $\mathbb{K}$ such that $\xi-\varphi_{n}$ and $\xi^{2}-\psi_{n}$ are linearly independent over $\mathbb{K}$ and

$$
\begin{gathered}
\max \left\{\limsup _{n \rightarrow \infty} \frac{1}{n} \log \left|\xi-\varphi_{n}\right|, \limsup _{n \rightarrow \infty} \frac{1}{n} \log \left|\xi^{2}-\psi_{n}\right|\right\} \leq-\varrho, \\
\limsup _{n \rightarrow \infty} \frac{1}{n} \mathrm{~h}\left(\left(1: \varphi_{n}: \psi_{n}\right)\right) \leq c
\end{gathered}
$$

for positive real numbers $\varrho$ and c. If

$$
\lambda:=\frac{1}{\delta}-\frac{c}{\varrho}>0,
$$

then $\xi$ is nonquadratic over $\mathbb{K}$, and

$$
\mu_{2, \mathbb{K}}(\xi) \leq \lambda^{-1}
$$

Proof. By applying Lemma 2.6 to $\xi$ and $\xi^{2}$, we see that $\xi$ is nonquadratic over $\mathbb{K}$. For any $\beta \in \mathbb{K}$ with $\mathrm{h}(\beta)$ sufficiently large,

$$
\log |\xi-\beta|>-\frac{1+\varepsilon}{\lambda} \mathrm{h}(\beta)>-\frac{2+\varepsilon}{\lambda} \mathrm{h}(\beta)
$$

by Lemma 2.2 with $\vartheta_{n}=\varphi_{n}$. For an arbitrary $\beta$ with $[\mathbb{K}(\beta): \mathbb{K}]=2$, let $p, q \in \mathbb{K}$ satisfy $p+q \beta+\beta^{2}=0$. Then

$$
2 \mathrm{~h}(\beta)=\mathrm{h}\left(\beta^{2}\right)=\mathrm{h}(p+q \beta) \leq \mathrm{h}((1: p: q))+\mathrm{h}(\beta)+\log 2,
$$

since $\max \{1, a+b x\} \leq 2 \max \{1, x\} \max \{1, a, b\}$ for any $a, b, x \geq 0$. If $\beta^{\prime}$ is the conjugate of $\beta$ over $\mathbb{K}$, then

$$
\mathrm{h}((1: p: q))=\mathrm{h}\left(\left(1: \beta \beta^{\prime}:-\left(\beta+\beta^{\prime}\right)\right)\right) \leq \mathrm{h}(\beta)+\mathrm{h}\left(\beta^{\prime}\right)+\log 2=2 \mathrm{~h}(\beta)+\log 2,
$$

since $\max \{1, x y, x+y\} \leq 2 \max \{x, 1\} \max \{y, 1\}$ for any $x, y \geq 0$. Thus $\mathrm{h}((1: p: q))$ is bounded from above and from below as follows:

$$
\mathrm{h}(\beta)-\log 2 \leq \mathrm{h}((1: p: q)) \leq 2 \mathrm{~h}(\beta)+\log 2 .
$$

Again by Lemma 2.6,

$$
\mu_{\mathbb{K}}\left(\left(1: \xi: \xi^{2}\right)\right) \leq \lambda^{-1},
$$


that is, by Definition 2.4, Remark 2.5 and formula (2.20),

$$
\log \frac{\left|p+q \xi+\xi^{2}\right|}{\max \{|q|, 1\}}>-\left(1+\frac{\varepsilon}{4}\right) \lambda^{-1} \mathrm{~h}((1: p: q))>-(2+\varepsilon) \lambda^{-1} \mathrm{~h}(\beta)
$$

for any $\beta$ with sufficiently large $\mathrm{h}(\beta)$. Furthermore, if $|\xi-\beta|<1$, which is not restrictive, then from

$$
p+q \xi+\xi^{2}=p+q \xi+\xi^{2}-\left(p+q \beta+\beta^{2}\right)=(\xi-\beta)(2 \xi+q+(\beta-\xi))
$$

it follows that

$$
\left|p+q \xi+\xi^{2}\right| \leq|\xi-\beta|(2|\xi|+|q|+1) \leq 4|\xi-\beta| \max \{1,|\xi|\} \max \{|q|, 1\} .
$$

Therefore, by (2.21) and (2.22),

$$
\log |\xi-\beta|+\log ^{+}|\xi|+2 \log 2>-(2+\varepsilon) \lambda^{-1} \mathrm{~h}(\beta) .
$$

Hence

$$
\log |\xi-\beta|>-2 \frac{1+\varepsilon}{\lambda} \mathrm{h}(\beta)
$$

for any $\beta$ with sufficiently large $\mathrm{h}(\beta)$.

As a consequence of Corollary 2.8 we obtain a proof of Remark 1 in [H2, p. 4567].

Corollary 2.9 (Hata $[\mathrm{H} 2])$. Let $\xi \in \mathbb{R}$ with $[\mathbb{Q}(\xi): \mathbb{Q}]>2$, and let $\left(p_{n}\right)_{n \in \mathbb{N}},\left(q_{n}\right)_{n \in \mathbb{N}}$ and $\left(r_{n}\right)_{n \in \mathbb{N}}$ be sequences of integers such that

$$
\lim _{n \rightarrow \infty} \frac{1}{n} \log \left|q_{n}\right|=\sigma
$$

and

$$
\max \left\{\limsup _{n \rightarrow \infty} \frac{1}{n} \log \left|q_{n} \xi-p_{n}\right|, \limsup _{n \rightarrow \infty} \frac{1}{n} \log \left|q_{n} \xi^{2}-r_{n}\right|\right\} \leq-\tau
$$

for positive real numbers $\sigma$ and $\tau$. Then

$$
\mu_{2}(\xi)=\mu_{2, \mathbb{Q}}(\xi) \leq 1+\frac{\sigma}{\tau} .
$$

Proof. Let $\varphi_{n}=p_{n} / q_{n}$ and $\psi_{n}=r_{n} / q_{n}$, and let $\mathbb{K}=\mathbb{Q}$. As in the proof of Corollary 2.3,

$$
\max \left\{\limsup _{n \rightarrow \infty} \frac{1}{n} \log \left|\xi-\varphi_{n}\right|, \limsup _{n \rightarrow \infty} \frac{1}{n} \log \left|\xi^{2}-\psi_{n}\right|\right\} \leq-(\sigma+\tau) .
$$

Moreover, $\mathrm{h}\left(\left(1: \varphi_{n}: \psi_{n}\right)\right) \leq \log \max \left\{\left|p_{n}\right|,\left|q_{n}\right|,\left|r_{n}\right|\right\}$, and

$$
p_{n}=q_{n} \xi\left(1-\frac{q_{n} \xi-p_{n}}{q_{n} \xi}\right) \quad \text { and } \quad r_{n}=q_{n} \xi^{2}\left(1-\frac{q_{n} \xi^{2}-r_{n}}{q_{n} \xi^{2}}\right)
$$


whence

$$
\lim _{n \rightarrow \infty} \frac{1}{n} \log \left|p_{n}\right|=\lim _{n \rightarrow \infty} \frac{1}{n} \log \left|q_{n}\right|=\lim _{n \rightarrow \infty} \frac{1}{n} \log \left|r_{n}\right|=\sigma .
$$

From Corollary 2.8 with $\lambda=1-\sigma /(\sigma+\tau)=\tau /(\sigma+\tau)$,

$$
\mu_{2}(\xi) \leq \lambda^{-1}=1+\frac{\sigma}{\tau}
$$

as required.

\section{Double integrals}

In the following sections, we shall combine the algebraic lemmas of Section 2 with the study of the asymptotics of linear forms in logarithms and of their coefficients, expressed by suitable generalizations of the double complex integrals introduced in [M]. Specifically, we extend the integrals of Marcovecchio [M] by taking the parameter $x$ therein to be a suitable algebraic number $\alpha \in \mathbb{C}$ with $\operatorname{deg} \alpha \geq 2$, or any one of its algebraic conjugates.

Let $h, j, k, l, m, q$ be positive integers satisfying

$$
h+j+q=k+l+m,
$$

and such that

$$
\begin{gathered}
l+k-j=h+q-m>0, \\
j+h-k=m+l-q>0, \\
k+m-h=q+j-l>0 .
\end{gathered}
$$

Let $x \in \mathbb{C} \backslash\{0\}$, and denote by $\operatorname{Arg} x$ the branch of the argument of $x$ such that $-\pi \leq \operatorname{Arg} x<\pi$. For any $\eta_{1}, \eta_{2} \in \mathbb{C}$ satisfying $\left|\eta_{1}\right|=\left|\eta_{2}\right|=1$ and

$$
\max \{0, \operatorname{Arg} x\}<\arg \eta_{1}<\arg \eta_{2}<2 \pi+\min \{0, \operatorname{Arg} x\},
$$

we define

$$
\begin{aligned}
I^{+}(x)= & I^{+}(h, j, k, l, m, q ; x) \\
= & x^{\max \{0, q-l, m-h\}}(1-x)^{k+l+m+1} \\
& \quad \times \int_{s=0}^{\eta_{1} \infty} \int_{t=0}^{\eta_{2} \infty} \frac{s^{h} t^{j}}{(1-s)^{l+k-j+1}(s-t)^{j+h-k+1}(t-x)^{k+m-h+1}} d t d s,
\end{aligned}
$$

where the notation for the limits of integration means that the integration paths for $s$ and $t$ are the half-lines going from zero to infinity through the points $\eta_{1}$ and $\eta_{2}$ respectively. Similarly, for any $\eta_{1}, \eta_{2}$ as above, we define

$$
\begin{aligned}
I^{-}(x)= & I^{-}(h, j, k, l, m, q ; x) \\
= & x^{\max \{0, q-l, m-h\}}(1-x)^{k+l+m+1} \\
& \quad \times \int_{s=0}^{\eta_{2} \infty} \int_{t=0}^{\eta_{1} \infty} \frac{s^{h} t^{j}}{(1-s)^{l+k-j+1}(s-t)^{j+h-k+1}(t-x)^{k+m-h+1}} d t d s .
\end{aligned}
$$


For $x \in \mathbb{C} \backslash\{1\}$, we also define the double contour integral

$$
\begin{aligned}
J(x)= & J(h, j, k, l, m, q ; x) \\
= & \frac{1}{(2 \pi i)^{2}} x^{\max \{0, q-l, m-h\}}(1-x)^{k+l+m+1} \\
& \quad \times \oint_{|s-x|=\varrho} \oint_{|t-x|=\varrho^{\prime}} \frac{s^{h} t^{j}}{(1-s)^{l+k-j+1}(s-t)^{j+h-k+1}(t-x)^{k+m-h+1}} d t d s,
\end{aligned}
$$

for any $\varrho$ and $\varrho^{\prime}$ satisfying $0<\varrho^{\prime}<\varrho<|1-x|$.

From the results in [M, Sections 2 and 3], we have the following facts.

(i) The integrals $I^{+}(x)$ and $I^{-}(x)$ are unchanged by replacing $\eta_{1}, \eta_{2}$ with $\eta_{1}^{\prime}, \eta_{2}^{\prime}$ respectively, for any $\eta_{1}^{\prime}$ and $\eta_{2}^{\prime}$ satisfying $\left|\eta_{1}^{\prime}\right|=\left|\eta_{2}^{\prime}\right|=1$ and

$$
\max \{0, \operatorname{Arg} x\}<\arg \eta_{1}^{\prime}<\arg \eta_{2}^{\prime}<2 \pi+\min \{0, \operatorname{Arg} x\} .
$$

Also, $I^{+}(x)$ and $I^{-}(x)$ converge absolutely, so that the integrations in $s$ and $t$ can be interchanged, and converge locally uniformly for $x$ in $\mathbb{C} \backslash\{0\}$. Thus $I^{+}(x)$ and $I^{-}(x)$ are analytic functions of $x$, in general with a branch point at $x=0$.

(ii) For $x \in \mathbb{R}^{+}$,

$$
\begin{gathered}
I^{+}(x)=\overline{I^{-}(x)}=P(x) \frac{1}{2} \log ^{2}(1 / x)-Q(x) \log (1 / x)+R(x) \\
+\pi i(P(x) \log (1 / x)-Q(x))
\end{gathered}
$$

for polynomials $P(x), Q(x), R(x) \in \mathbb{Q}[x]$, whence

$$
\begin{aligned}
& \frac{I^{+}(x)+I^{-}(x)}{2}=P(x) \frac{1}{2} \log ^{2}(1 / x)-Q(x) \log (1 / x)+R(x), \\
& \frac{I^{+}(x)-I^{-}(x)}{2 \pi i}=P(x) \log (1 / x)-Q(x) .
\end{aligned}
$$

Moreover, for $x \in \mathbb{R}^{+} \backslash\{1\}$,

$$
J(x)=P(x) .
$$

By analytic continuation, (3.6)-(3.8) hold for any $x \in \mathbb{C} \backslash\{0,1\}$, where in (3.6) and (3.7) the $\operatorname{logarithm}$ is defined by $\log z=\log |z|+i \arg z$ with $-\pi<\arg z \leq \pi$.

(iii) There exist explicitly given positive integers $M, N, \gamma_{1}, \gamma_{2}$, with $N \leq M$ and $\gamma_{1} \leq \gamma_{2}$, depending only on $h, j, k, l, m, q$, such that the polynomials $P(x), Q(x)$, $R(x)$ in (ii) satisfy

$$
\begin{aligned}
& \max \{\operatorname{deg} P, \operatorname{deg} Q\} \leq \gamma_{1}, \quad \operatorname{deg} R \leq \gamma_{2}, \\
& P(x), d_{M} Q(x), d_{M} d_{N} R(x) \in \mathbb{Z}[x],
\end{aligned}
$$

where $d_{r}$ denotes the least common multiple of the integers from 1 to $r$ for any positive integer $r$. Moreover, the integrals $I^{+}(x), I^{-}(x), J(x)$ and the integers $M$, $N, \gamma_{1}, \gamma_{2}$ are all invariant under the action on $\{h, j, k, l, m, q\}$ of the permutations

$$
\boldsymbol{\sigma}=(h l)(j k)(m q) \quad \text { and } \quad \boldsymbol{\tau}=(h k)(j m)(l q) .
$$


(iv) For suitable choices of $h, j, k, l, m, q$ not all equal, the integer coefficients of the polynomials (3.10) have a large common divisor. More precisely, let $I_{n}^{+}(x)$, $I_{n}^{-}(x), J_{n}(x)=P_{n}(x), Q_{n}(x), R_{n}(x)$ denote the integrals and polynomials in (ii) with $h, j, k, l, m, q$ replaced by $h n, j n, k n, l n, m n, q n$ respectively, where $h, j, k$, $l, m, q$ are fixed and $n$ is any positive integer. Then the integers $M, N, \gamma_{1}, \gamma_{2}$ in (iii) are changed to $M n, N n, \gamma_{1} n, \gamma_{2} n$, respectively. In particular, (3.9) becomes

$$
\max \left\{\operatorname{deg} P_{n}, \operatorname{deg} Q_{n}\right\} \leq \gamma_{1} n, \quad \operatorname{deg} R_{n} \leq \gamma_{2} n .
$$

For any $n$, there exists a positive integer $D_{n}$ such that $D_{n} \mid d_{M n}$ and

$$
\frac{D_{n}}{d_{M n}} P_{n}(x), D_{n} Q_{n}(x), D_{n} d_{N n} R_{n}(x) \in \mathbb{Z}[x] .
$$

Moreover,

$$
\lim _{n \rightarrow \infty} \frac{1}{n} \log D_{n}=M-\int_{\Omega} d \psi(z),
$$

where $\psi(z)=\Gamma^{\prime}(z) / \Gamma(z)$ is the logarithmic derivative of the Euler gammafunction, and $\Omega$ is a suitable union of subintervals of $[0,1)$ with rational endpoints depending only on $h, j, k, l, m, q$ (for the precise definitions of the integers $\gamma_{1}$ and $\gamma_{2}$ see [M, formula (15)] (denoted there by $\gamma$ and $\delta$ ), for the integers $M, N$ and $D_{n}$ see [M, p. 173] and for the set $\Omega$ see [M, p. 180]).

\section{Main theorems}

In this section we show how to apply the algebraic lemmas of Section 2 to the double integrals $I_{n}^{+}, I_{n}^{-}$and $J_{n}$ of Section 3, in order to obtain $\mathbb{Q}(\alpha)$-irrationality and $\mathbb{Q}(\alpha)$-nonquadraticity measures for the logarithms of some algebraic numbers $\alpha \in \mathbb{C}$. We shall use Hata's $\mathbb{C}^{2}$-saddle point method [H2] for the study of the asymptotic behaviours of the double integrals as $n \rightarrow \infty$. However, since the technical details in the various applications of the $\mathbb{C}^{2}$-saddle point method strongly depend on $\alpha$, we prefer to postpone such applications to the next sections, for several different choices of $\alpha$. Here we prove the desired upper bounds for $\mu_{\mathbb{Q}(\alpha)}(\log \alpha)$ and $\mu_{2, \mathbb{Q}(\alpha)}(\log \alpha)$ subject to conditional assumptions on the asymptotics of the double integrals, which will be justified in the next sections.

Let $\alpha \in \mathbb{C} \backslash\{0,1\}$ be an algebraic number with $\operatorname{deg} \alpha=\Delta$, and let $\alpha_{1}=\alpha, \alpha_{2}, \ldots, \alpha_{\Delta}$ be its algebraic conjugates. Let

$$
a_{0}\left(x-\alpha_{1}\right) \cdots\left(x-\alpha_{\Delta}\right)
$$

be the minimal polynomial of $\alpha$ in $\mathbb{Z}[x]$, with $a_{0}>0$. Suppose that there exist positive constants $H, K_{1}, \ldots, K_{\Delta}$ such that

$$
\begin{gathered}
\max \left\{\limsup _{n \rightarrow \infty} \frac{1}{n} \log \left|I_{n}^{+}(\alpha)\right|, \limsup _{n \rightarrow \infty} \frac{1}{n} \log \left|I_{n}^{-}(\alpha)\right|\right\} \leq-H, \\
\lim _{n \rightarrow \infty} \frac{1}{n} \log \left|J_{n}(\alpha)\right|=K_{1},
\end{gathered}
$$


and

$\max \left\{\limsup _{n \rightarrow \infty} \frac{1}{n} \log \left|I_{n}^{+}\left(\alpha_{d}\right)\right|, \limsup _{n \rightarrow \infty} \frac{1}{n} \log \left|I_{n}^{-}\left(\alpha_{d}\right)\right|, \limsup _{n \rightarrow \infty} \frac{1}{n} \log \left|J_{n}\left(\alpha_{d}\right)\right|\right\} \leq K_{d}$

when $d=2, \ldots, \Delta$. According to $(2.5)$, let

$$
\delta(\alpha)= \begin{cases}\Delta & \text { if } \alpha \in \mathbb{R} \\ \frac{1}{2} \Delta & \text { if } \alpha \notin \mathbb{R} .\end{cases}
$$

Let $M, N, \gamma_{1}, \gamma_{2}$ be the integers in (3.11)-(3.13). Finally, let

$$
\begin{aligned}
& \lambda(\alpha)=\frac{1}{\delta(\alpha)}-\frac{1}{H+K_{1}}\left(M-\int_{\Omega} d \psi(z)+\frac{1}{\Delta}\left(\sum_{d=1}^{\Delta} K_{d}+\gamma_{1} \log a_{0}\right)\right), \\
& \lambda_{2}(\alpha)=\frac{1}{\delta(\alpha)}-\frac{1}{H+K_{1}}\left(M+N-\int_{\Omega} d \psi(z)+\frac{1}{\Delta}\left(\sum_{d=1}^{\Delta} K_{d}+\gamma_{2} \log a_{0}\right)\right),
\end{aligned}
$$

with $\int_{\Omega} d \psi(z)$ as in (3.13), and let $\log \alpha=\log |\alpha|+i \arg \alpha$, with $-\pi<\arg \alpha \leq \pi$, be the principal value of the logarithm.

Under the assumptions above, we prove the following theorems.

THEOREM 4.1. If $\lambda(\alpha)>0$, then

$$
\mu_{\mathbb{Q}(\alpha)}(\log \alpha) \leq \lambda(\alpha)^{-1} .
$$

THeorem 4.2. If $\lambda_{2}(\alpha)>0$, then

$$
\mu_{2, \mathbb{Q}(\alpha)}(\log \alpha) \leq \lambda_{2}(\alpha)^{-1} .
$$

Proof of Theorem 4.1. Let $P_{n}, Q_{n}$ and $R_{n}$ be the polynomials in (3.11) and (3.12). By (3.8) and (4.2), $P_{n}(\alpha)=J_{n}(\alpha) \neq 0$ for any sufficiently large $n$. Thus we can apply Lemma 2.2 with $\mathbb{K}=\mathbb{Q}(\alpha), \xi=\log (1 / \alpha)$ and $\vartheta_{n}=Q_{n}(\alpha) / P_{n}(\alpha)$. Hence, by (3.7),

$$
\xi-\vartheta_{n}=\frac{I_{n}^{+}(\alpha)-I_{n}^{-}(\alpha)}{2 \pi i J_{n}(\alpha)} .
$$

Therefore

$$
\left|\xi-\vartheta_{n}\right| \leq \frac{\max \left\{\left|I_{n}^{+}(\alpha)\right|,\left|I_{n}^{-}(\alpha)\right|\right\}}{\pi\left|J_{n}(\alpha)\right|}
$$

For $n \rightarrow \infty$ we obtain, by (4.2),

$$
\limsup _{n \rightarrow \infty} \frac{1}{n} \log \left|\xi-\vartheta_{n}\right| \leq-\left(H+K_{1}\right)
$$


so that the constant $\varrho$ in Lemma 2.2 can be given by

$$
\varrho=H+K_{1} .
$$

As for the constant $c$ in Lemma 2.2, we get by (2.1)

$$
\frac{1}{n} \mathrm{~h}\left(\vartheta_{n}\right)=\frac{1}{n \Delta} \sum_{v \in \mathcal{M}_{\mathrm{Q}(\alpha)}} \eta_{v} \log ^{+}\left|\frac{D_{n} Q_{n}(\alpha)}{D_{n} P_{n}(\alpha)}\right|_{v}
$$

where $D_{n}$ is the integer in (3.12) and (3.13).

Applying the product formula (2.3) to $D_{n} P_{n}(\alpha)$

$$
\begin{aligned}
\frac{1}{n} \mathrm{~h}\left(\vartheta_{n}\right) & =\frac{1}{n \Delta} \sum_{v \in \mathcal{M}_{\mathbb{Q}(\alpha)}} \eta_{v}\left(\log ^{+}\left|\frac{D_{n} Q_{n}(\alpha)}{D_{n} P_{n}(\alpha)}\right|_{v}+\log \left|D_{n} P_{n}(\alpha)\right|_{v}\right) \\
& =\frac{1}{\Delta} \sum_{v \in \mathcal{M}_{\mathbb{Q}(\alpha)}} \eta_{v} \frac{1}{n} \log \max \left\{\left|D_{n} P_{n}(\alpha)\right|_{v},\left|D_{n} Q_{n}(\alpha)\right|_{v}\right\} .
\end{aligned}
$$

For the Archimedean places,

$$
\begin{aligned}
& \frac{1}{\Delta} \sum_{v \mid \infty} \eta_{v} \frac{1}{n} \log \max \left\{D_{n}\left|P_{n}(\alpha)\right|_{v}, D_{n}\left|Q_{n}(\alpha)\right|_{v}\right\} \\
& =\left(\frac{1}{n} \log D_{n}\right) \frac{1}{\Delta} \sum_{v \mid \infty} \eta_{v}+\frac{1}{\Delta} \sum_{v \mid \infty} \eta_{v} \frac{1}{n} \log \max \left\{\left|P_{n}(\alpha)\right|_{v},\left|Q_{n}(\alpha)\right|_{v}\right\} \\
& =\frac{1}{n} \log D_{n}+\frac{1}{\Delta} \sum_{d=1}^{\Delta} \frac{1}{n} \log \max \left\{\left|P_{n}\left(\alpha_{d}\right)\right|,\left|Q_{n}\left(\alpha_{d}\right)\right|\right\} .
\end{aligned}
$$

By (3.7), the last quantity does not exceed

$$
\frac{1}{n} \log D_{n}+\frac{1}{\Delta} \sum_{d=1}^{\Delta} \frac{1}{n} \log \max \left\{\left|P_{n}\left(\alpha_{d}\right)\right|,\left|P_{n}\left(\alpha_{d}\right) \log \left(1 / \alpha_{d}\right)\right|+\frac{1}{2 \pi}\left|I_{n}^{+}\left(\alpha_{d}\right)\right|+\frac{1}{2 \pi}\left|I_{n}^{-}\left(\alpha_{d}\right)\right|\right\} .
$$

Hence, by (3.8), (3.13), (4.2) and (4.3),

$$
\begin{aligned}
\limsup _{n \rightarrow \infty} \frac{1}{\Delta} \sum_{v \mid \infty} \eta_{v} \frac{1}{n} \log \max \left\{D_{n}\left|P_{n}(\alpha)\right|_{v}, D_{n}\left|Q_{n}(\alpha)\right|_{v}\right\} \\
\leq M-\int_{\Omega} d \psi(z)+\frac{1}{\Delta} \sum_{d=1}^{\Delta} \max \left\{\limsup _{n \rightarrow \infty} \frac{1}{n} \log \left|J_{n}\left(\alpha_{d}\right)\right|,\right. \\
\left.\quad \limsup _{n \rightarrow \infty} \frac{1}{n} \log \left|I_{n}^{+}\left(\alpha_{d}\right)\right|, \limsup _{n \rightarrow \infty} \frac{1}{n} \log \left|I_{n}^{-}\left(\alpha_{d}\right)\right|\right\} \\
\leq M-\int_{\Omega} d \psi(z)+\frac{1}{\Delta} \sum_{d=1}^{\Delta} K_{d} .
\end{aligned}
$$


By (3.11) and (3.12), we have $D_{n} P_{n}(\alpha), D_{n} Q_{n}(\alpha) \in \mathbb{Z}[\alpha]$, with

$$
\max \left\{\operatorname{deg} P_{n}, \operatorname{deg} Q_{n}\right\} \leq \gamma_{1} n .
$$

Hence for any ultrametric place $v$,

$$
\max \left\{\left|D_{n} P_{n}(\alpha)\right|_{v},\left|D_{n} Q_{n}(\alpha)\right|_{v}\right\} \leq\left(\max \left\{1,|\alpha|_{v}\right\}\right)^{\gamma_{1} n} .
$$

It follows that

$$
\frac{1}{\Delta} \sum_{v \nmid \infty} \eta_{v} \frac{1}{n} \log \max \left\{\left|D_{n} P_{n}(\alpha)\right|_{v},\left|D_{n} Q_{n}(\alpha)\right|_{v}\right\} \leq \frac{\gamma_{1}}{\Delta} \sum_{v \nmid \infty} \eta_{v} \log ^{+}|\alpha|_{v} .
$$

It is well known that, since (4.1) is the minimal polynomial of $\alpha$,

$$
\mathrm{h}(\alpha)=\frac{1}{\Delta}\left(\log a_{0}+\sum_{d=1}^{\Delta} \log ^{+}\left|\alpha_{d}\right|\right)=\frac{1}{\Delta}\left(\log a_{0}+\sum_{\nu \mid \infty} \eta_{v} \log ^{+}|\alpha|_{v}\right) .
$$

Combining with (2.1), this yields

$$
\sum_{v \nmid \infty} \eta_{v} \log ^{+}|\alpha|_{v}=\log a_{0} .
$$

Therefore

$$
\frac{1}{\Delta} \sum_{v \nmid \infty} \eta_{v} \frac{1}{n} \log \max \left\{\left|D_{n} P_{n}(\alpha)\right|_{v},\left|D_{n} Q_{n}(\alpha)\right|_{v}\right\} \leq \frac{\gamma_{1} \log a_{0}}{\Delta} .
$$

As $n \rightarrow \infty$ we obtain, by (4.8), (4.9) and (4.11),

$$
\limsup _{n \rightarrow \infty} \frac{1}{n} \mathrm{~h}\left(\vartheta_{n}\right) \leq M-\int_{\Omega} d \psi(z)+\frac{1}{\Delta} \sum_{d=1}^{\Delta} K_{d}+\frac{\gamma_{1} \log a_{0}}{\Delta} .
$$

Thus we may take the constant $c$ in Lemma 2.2 to be

$$
c=M-\int_{\Omega} d \psi(z)+\frac{1}{\Delta}\left(\sum_{d=1}^{\Delta} K_{d}+\gamma_{1} \log a_{0}\right) .
$$

By (4.7) and (4.12), the desired conclusion follows from Lemma 2.2.

Proof of Theorem 4.2. Here we apply Corollary 2.8 with $\mathbb{K}=\mathbb{Q}(\alpha), \xi=\log (1 / \alpha)$, $\varphi_{n}=Q_{n}(\alpha) / P_{n}(\alpha)$, and $\psi_{n}=2 R_{n}(\alpha) / P_{n}(\alpha)$. As with (4.6),

$$
\limsup _{n \rightarrow \infty} \frac{1}{n} \log \left|\xi-\varphi_{n}\right| \leq-\left(H+K_{1}\right) \text {. }
$$

From (3.6) and (3.7) with $x=\alpha$,

$$
\frac{I_{n}^{+}(\alpha)-I_{n}^{-}(\alpha)}{2 \pi i} \log (1 / \alpha)-\frac{I_{n}^{+}(\alpha)+I_{n}^{-}(\alpha)}{2}=P_{n}(\alpha) \frac{1}{2} \log ^{2}(1 / \alpha)-R_{n}(\alpha) .
$$


Thus, by (3.8),

$$
\xi^{2}-\psi_{n}=\frac{(\log (1 / \alpha)-\pi i) I_{n}^{+}(\alpha)-(\log (1 / \alpha)+\pi i) I_{n}^{-}(\alpha)}{\pi i J_{n}(\alpha)},
$$

whence, by (4.2),

$$
\limsup _{n \rightarrow \infty} \frac{1}{n} \log \left|\xi^{2}-\psi_{n}\right| \leq-\left(H+K_{1}\right) .
$$

Since the Weil height $\mathrm{h}\left(\left(1: \varphi_{n}: \psi_{n}\right)\right)$ is independent of the choice of the homogeneous coordinates $1, \varphi_{n}, \psi_{n}$,

$$
\mathrm{h}\left(\left(1: \varphi_{n}: \psi_{n}\right)\right)=\mathrm{h}\left(\left(D_{n} d_{N n} P_{n}(\alpha): D_{n} d_{N n} Q_{n}(\alpha): 2 D_{n} d_{N n} R_{n}(\alpha)\right)\right)
$$

whence, by (2.11),

$$
\begin{aligned}
& \frac{1}{n} \mathrm{~h}\left(\left(1: \varphi_{n}: \psi_{n}\right)\right) \\
& \quad=\frac{1}{\Delta} \sum_{v \in \mathcal{M}_{\mathrm{Q}(\alpha)}} \eta_{v} \frac{1}{n} \log \max \left\{\left|D_{n} d_{N n} P_{n}(\alpha)\right|_{v},\left|D_{n} d_{N n} Q_{n}(\alpha)\right|_{v},\left|2 D_{n} d_{N n} R_{n}(\alpha)\right|_{v}\right\},
\end{aligned}
$$

similarly to (4.8). For the Archimedean places we now have, using (3.6) and (3.7),

$$
\begin{aligned}
\frac{1}{\Delta} \sum_{v \mid \infty} & \eta_{v} \frac{1}{n} \log \max \left\{D_{n} d_{N n}\left|P_{n}(\alpha)\right|_{v}, D_{n} d_{N n}\left|Q_{n}(\alpha)\right|_{v}, 2 D_{n} d_{N n}\left|R_{n}(\alpha)\right|_{v}\right\} \\
= & \frac{1}{n} \log D_{n}+\frac{1}{n} \log d_{N n}+\frac{1}{\Delta} \sum_{d=1}^{\Delta} \frac{1}{n} \log \max \left\{\left|P_{n}\left(\alpha_{d}\right)\right|,\left|Q_{n}\left(\alpha_{d}\right)\right|, 2\left|R_{n}\left(\alpha_{d}\right)\right|\right\} \\
\leq & \frac{1}{n} \log D_{n}+\frac{1}{n} \log d_{N n} \\
& +\frac{1}{\Delta} \sum_{d=1}^{\Delta} \frac{1}{n} \log \max \left\{\left|P_{n}\left(\alpha_{d}\right)\right|,\left|P_{n}\left(\alpha_{d}\right) \log \left(1 / \alpha_{d}\right)\right|+\frac{1}{2 \pi}\left|I_{n}^{+}\left(\alpha_{d}\right)\right|+\frac{1}{2 \pi}\left|I_{n}^{-}\left(\alpha_{d}\right)\right|,\right. \\
& \left.\left|P_{n}\left(\alpha_{d}\right) \log ^{2}\left(1 / \alpha_{d}\right)\right|+\left(\frac{1}{\pi}\left|\log \left(1 / \alpha_{d}\right)\right|+1\right)\left(\left|I_{n}^{+}\left(\alpha_{d}\right)\right|+\left|I_{n}^{-}\left(\alpha_{d}\right)\right|\right)\right\} .
\end{aligned}
$$

By the prime number theorem, $\lim _{n \rightarrow \infty}(1 / n) \log d_{N n}=N$. Hence, by (3.8), (3.13), (4.2) and (4.3),

$$
\begin{aligned}
& \limsup _{n \rightarrow \infty} \frac{1}{\Delta} \sum_{v \mid \infty} \eta_{v} \frac{1}{n} \log \max \left\{D_{n} d_{N n}\left|P_{n}(\alpha)\right|_{v}, D_{n} d_{N n}\left|Q_{n}(\alpha)\right|_{v}, 2 D_{n} d_{N n}\left|R_{n}(\alpha)\right|_{v}\right\} \\
& \leq M+N-\int_{\Omega} d \psi(z)+\frac{1}{\Delta} \sum_{d=1}^{\Delta} K_{d} .
\end{aligned}
$$


As in the proof of Theorem 4.1, by (3.11), (3.12) and (4.10),

$$
\begin{aligned}
& \frac{1}{\Delta} \sum_{v \nmid \infty} \eta_{v} \frac{1}{n} \log \max \left\{\left|D_{n} d_{N n} P_{n}(\alpha)\right|_{v},\left|D_{n} d_{N n} Q_{n}(\alpha)\right|_{v},\left|2 D_{n} d_{N n} R_{n}(\alpha)\right|_{v}\right\} \\
& \leq \frac{\gamma_{2}}{\Delta} \sum_{v \nmid \infty} \eta_{v} \log ^{+}|\alpha|_{v}=\frac{\gamma_{2} \log a_{0}}{\Delta} .
\end{aligned}
$$

Hence we may now take the constants $\varrho$ and $c$ to be given by

$$
\varrho=H+K_{1}, \quad c=M+N-\int_{\Omega} d \psi(z)+\frac{1}{\Delta}\left(\sum_{d=1}^{\Delta} K_{d}+\gamma_{2} \log a_{0}\right) .
$$

Then the conclusion follows from Corollary 2.8 .

\section{5. $\mathbb{C}^{2}$-saddle point method}

In several interesting cases, the best constants $H, K_{1}, \ldots, K_{\Delta}$ for Theorems 4.1 and 4.2 can be computed by applying Hata's $\mathbb{C}^{2}$-saddle point method [H2] to the double integrals $I_{n}^{+}(x), I_{n}^{-}(x)$ and $J_{n}(x)$ defined in Section 3(iv), independently for every $x=\alpha, \alpha_{2}, \ldots, \alpha_{\Delta}$. For every choice of $x$, the exact asymptotic behaviour, as $n \rightarrow \infty$, of each of $I_{n}^{+}(x), I_{n}^{-}(x)$ and $J_{n}(x)$ depends on the value of the function

$$
f(s, t):=\frac{s^{h} t^{j}}{(1-s)^{l+k-j}(s-t)^{j+h-k}(t-x)^{k+m-h}}
$$

at one of its stationary points in $\mathbb{C}^{2}$, that is, at a solution of the system

$$
\frac{\partial f}{\partial s}=\frac{\partial f}{\partial t}=0, \quad f(s, t) \neq 0 .
$$

Writing $\partial \log f / \partial s=0$ and $\partial \log f / \partial t=0$, and using (3.1), the system (5.1) becomes

$$
\left\{\begin{array}{l}
l s^{2}-(j-k) s+(m-q) s t-h t=0 \\
m t^{2}-(h-k) x t+(l-q) s t-j x s=0
\end{array}\right.
$$

The first step in applying the $\mathbb{C}^{2}$-saddle point method for each of the double integrals $I_{n}^{+}(x), I_{n}^{-}(x)$ and $J_{n}(x)$ (see [H2, Hypothesis A, p. 4559]) is to solve either the equation $\partial f / \partial s=0$ with respect to $s$, thus expressing $s$ as a holomorphic function $S(t)$ of $t$ in a suitable open region $D$ of the $t$-plane, or, interchanging the roles of $s$ and $t$, to solve the equation $\partial f / \partial t=0$ with respect to $t$. Since the parameter $x$ does not appear in the first equation (5.2), it is convenient to solve $\partial f / \partial s=0$ with respect to $s$, thus obtaining a function $s=S(t)$ independent of $x$.

For convenience, as in [M], besides (3.1) and (3.2) we choose the exponents in the function $f(s, t)$ to satisfy the condition

$$
0<k=q<h=l<j=m .
$$


By (5.3), the system (5.2) is

$$
\left\{\begin{array}{l}
h s^{2}-(j-k) s+(j-k) s t-h t=0 \\
j t^{2}-(h-k) x t+(h-k) s t-j x s=0 .
\end{array}\right.
$$

The first equation (5.4) can be written as

$$
t=T(s):=s \frac{h s-(j-k)}{h-(j-k) s},
$$

whence $T(s) T(1 / s)=1$, so that the inverse of (5.5) is a two-valued function $s$ of $t$ with branch points at

$$
t=\tau_{ \pm}:=\frac{(j-k)^{2}-2 h^{2} \pm 2 h \sqrt{h^{2}-(j-k)^{2}}}{(j-k)^{2}},
$$

corresponding to the solutions

$$
\sigma_{ \pm}:=\frac{h \pm \sqrt{h^{2}-(j-k)^{2}}}{j-k}
$$

of $d T / d s=0$, precisely such that

$$
\tau_{+}=T\left(\sigma_{-}\right) \quad \text { and } \quad \tau_{-}=T\left(\sigma_{+}\right)
$$

Since, by (3.2) and (5.3), $h+k-j=l+k-j>0$ and $j-k>0$, the values $\sigma_{+}$and $\sigma_{-}$ are real and distinct, and satisfy

$$
0<\sigma_{-}<\sigma_{+} \text {and } \sigma_{+} \sigma_{-}=1
$$

Similarly

$$
\tau_{-}<\tau_{+}<0 \text { and } \quad \tau_{+} \tau_{-}=1
$$

Standard computations show that the function $t=T(s)$ in (5.5) maps both the upper and the lower half-circumference of diameter $\left[\sigma_{+}, \sigma_{-}\right]$onto the real interval $\left[\tau_{-}, \tau_{+}\right]$. Thus it is easily seen that each of the open regions $C_{1}, C_{2}$ of the $s$-plane defined by

$$
\begin{aligned}
& C_{1}:=\left\{\operatorname{Im}(s)>0 \text { and }\left|s-\frac{h}{j-k}\right|>\frac{\sqrt{h^{2}-(j-k)^{2}}}{j-k}\right\}, \\
& C_{2}:=\left\{\operatorname{Im}(s)<0 \text { and }\left|s-\frac{h}{j-k}\right|<\frac{\sqrt{h^{2}-(j-k)^{2}}}{j-k}\right\}
\end{aligned}
$$

is bijectively transformed by $T(s)$ onto the lower $t$-half-plane

$$
D_{1}:=\{\operatorname{Im}(t)<0\},
$$


and similarly each of

$$
\begin{aligned}
& C_{3}:=\left\{\operatorname{Im}(s)>0 \text { and }\left|s-\frac{h}{j-k}\right|<\frac{\sqrt{h^{2}-(j-k)^{2}}}{j-k}\right\}, \\
& C_{4}:=\left\{\operatorname{Im}(s)<0 \text { and }\left|s-\frac{h}{j-k}\right|>\frac{\sqrt{h^{2}-(j-k)^{2}}}{j-k}\right\}
\end{aligned}
$$

is bijectively transformed by $T(s)$ onto the upper $t$-half-plane

$$
D_{2}:=\{\operatorname{Im}(t)>0\} .
$$

Hence the function $s=S(t)$, holomorphic in an open region $D$, to be used in the $\mathbb{C}^{2}$ saddle point method can be chosen as the inverse of $t=T(s)$ either on $D=D_{1}$ with values in one of the regions $C_{1}$ or $C_{2}$, or on $D=D_{2}$ with values in $C_{3}$ or $C_{4}$, or on the $t$-plane deprived of $\left(-\infty, \tau_{-}\right] \cup\left[\tau_{+},+\infty\right)$ with values in one of the $s$-half-planes $\{\operatorname{Im}(s)>0\}$ or $\{\operatorname{Im}(s)<0\}$, and so on.

In order to find the stationary points of $f(s, t)$, we solve the system (5.4). We eliminate $t$ by substituting $T(s)$ for $t$ in the second equation (5.4). Dividing by $(j+h-k) s$, we obtain the cubic equation in $s$ :

$$
\begin{aligned}
& h k s^{3}-((j-h)(j-k) x+h(j-h)+k(j-k)) s^{2} \\
& \quad+((j-h)(j-k)+(h(j-h)+k(j-k)) x) s-h k x=0 .
\end{aligned}
$$

If we solve (5.6) with respect to $x$ we get

$$
x=X(s):=s \frac{h s-(j-k)}{h-(j-k) s} \frac{k s-(j-h)}{k-(j-h) s}=T(s) \frac{k s-(j-h)}{k-(j-h) s},
$$

whence $X(s) X(1 / s)=1$. Let $s=s_{1}, s_{2}, s_{3}$ be the roots of (5.6), and let $t_{v}=T\left(s_{v}\right)$ (where $v=1,2,3$ ), so that the stationary points of $f(s, t)$ (depending on $x$ ) with $f(s, t) \neq 0$ are $\left(s_{1}, t_{1}\right),\left(s_{2}, t_{2}\right)$ and $\left(s_{3}, t_{3}\right)$.

For the applications of the $\mathbb{C}^{2}$-saddle point method for every choice of $x$ and for each of the double integrals $I_{n}^{+}(x), I_{n}^{-}(x)$ and $J_{n}(x)$, we take two steps.

Step (i). Transform the integration path for $t$, applying Cauchy's theorem, into a new integration path $\gamma$ passing through one of $t_{1}, t_{2}, t_{3}\left(\right.$ say, $\left.t_{*}\right)$, so that $S\left(t_{*}\right)=s_{*}$ and

$$
\max _{t \in \gamma}|f(S(t), t)|=\left|f\left(s_{*}, t_{*}\right)\right|
$$

and the maximum on $\gamma$ is attained only at $t=t_{*}$ (see [H2, Hypothesis C]).

Step (ii). For any $t \in \gamma$, transform the integration path for $s$, again applying Cauchy's theorem, into a new integration path $\delta_{t}$ (in general depending on $t$ ) passing through $S(t)$, so that

$$
\max _{s \in \delta_{t}}|f(s, t)|=|f(S(t), t)|,
$$

and the maximum on $\delta_{t}$ is attained only at $s=S(t)$ (see [H2, Hypothesis E]). 
Then, by [H2, formula (1.9)],

$$
\lim _{n \rightarrow \infty} \frac{1}{n} \log \left|\int_{\gamma} d t \int_{\delta_{t}}(f(s, t))^{n} \frac{d s}{(1-s)(s-t)(t-x)}\right|=\log \left|f\left(s_{*}, t_{*}\right)\right| .
$$

In the next section we follow the strategy above for several different choices of $x$, and for each of $I_{n}^{+}(x), I_{n}^{-}(x)$ and $J_{n}(x)$.

\section{Numerical results}

6.1. We begin with some applications of Theorem 4.1, in all of which we choose the exponents in the function $f(s, t)$ as in [M, proof of Theorem 1.1], that is,

$$
k=q=4, \quad h=l=5, \quad j=m=6 .
$$

These choices yield

$$
M=7, \quad \gamma_{1}=11, \quad \int_{\Omega} d \psi(z)=4.99510233 \ldots
$$

(see [M, p. 181]).

For every choice of the algebraic number $\alpha \in \mathbb{C} \backslash\{0,1\}$, we find the constants $H$ and $K_{1}$ in (4.2) by applying the $\mathbb{C}^{2}$-saddle point method for $x=\alpha$, and the constants $K_{2}, \ldots, K_{\Delta}$ in (4.3) by applying the $\mathbb{C}^{2}$-saddle point method for $x=\alpha_{2}, \ldots, \alpha_{\Delta}$ (the algebraic conjugates of $\alpha$ ). We discuss in detail the first example, that is, $\alpha=1 / \sqrt{2}$, and we only sketch the discussion of the other examples.

(i) $\alpha=1 / \sqrt{2}$. By (4.1) and (4.4), we have $\delta(\alpha)=\Delta=2$ and $a_{0}=2$.

For $x=\alpha=1 / \sqrt{2}$, the roots of the cubic equation (5.6) are

$$
\begin{aligned}
& s_{1}=0.93310203 \ldots, \\
& s_{2}=-0.10619567 \ldots+i 0.86401660 \ldots, \\
& s_{3}=\overline{s_{2}} .
\end{aligned}
$$

The corresponding values $t_{v}=T\left(s_{v}\right)$ are

$$
\begin{aligned}
& t_{1}=0.79366780 \ldots, \\
& t_{2}=-0.44713001 \ldots-i 0.65579055 \ldots, \\
& t_{3}=\overline{t_{2}} .
\end{aligned}
$$

This yields

$$
\begin{aligned}
& \log \left|f\left(s_{1}, t_{1}\right)\right|=32.40667885 \ldots, \\
& \log \left|f\left(s_{2}, t_{2}\right)\right|=\log \left|f\left(s_{3}, t_{3}\right)\right|=-7.61520924 \ldots
\end{aligned}
$$

We have $I_{n}^{+}(1 / \sqrt{2})=\overline{I_{n}^{-}(1 / \sqrt{2})}$. According to (3.3), the initial integration paths in $s$ and $t$ for $I_{n}^{+}(1 / \sqrt{2})$ are the half-lines $\left(0, \eta_{1} \infty\right)$ and $\left(0, \eta_{2} \infty\right)$ respectively, for any $\eta_{1}, \eta_{2} \in \mathbb{C}$ 
satisfying $\left|\eta_{1}\right|=\left|\eta_{2}\right|=1$ and $0<\arg \eta_{1}<\arg \eta_{2}<2 \pi$. With the notation of Section 5, we take the function $s=S(t)$ for $t \in D=D_{1}=\{\operatorname{Im}(t)<0\}$ with values in $C_{1}$, and we choose the modified integration paths $\gamma$ for $t$ and $\delta=\delta_{t}$ for $s$, described in Steps (i) and (ii) in Section 5, as in [M, Section 5], that is,

$$
\delta=\mathbb{R}^{+} s_{2}=\left\{\lambda s_{2} \mid \lambda>0\right\}
$$

and

$$
\gamma=T(\delta)=\left\{\lambda s_{2} \frac{h \lambda s_{2}-(j-k)}{h-(j-k) \lambda s_{2}} \mid \lambda>0\right\} .
$$

Note that, in this case, the integration path $\delta$ for $s$ is independent of $t \in \gamma$. A discussion very similar to [M, pp. 179-180] shows that (5.8) and (5.9) hold. By (5.10) we conclude that the constant $H$ in (4.2) is

$$
H=-\log \left|f\left(s_{2}, t_{2}\right)\right|-(j-h) \log |x|-(h+j+k) \log |1-x|=26.38099049 \ldots,
$$

in accordance with [M, formula (43)].

According to (3.5), the initial integration contours in $s$ and $t$ for $J_{n}(1 / \sqrt{2})$ are the circles centred at $1 / \sqrt{2}$ with radii $\varrho$ and $\varrho^{\prime}$ respectively, where $0<\varrho^{\prime}<\varrho<1-1 / \sqrt{2}$. We now take $s=S(t)$ on the $t$-plane deprived of $\left[\tau_{-}, \tau_{+}\right]$with values in the punctured disc

$$
0<\left|s-\frac{h}{j-k}\right|<\frac{\sqrt{h^{2}-(j-k)^{2}}}{j-k},
$$

and we choose $\varrho^{\prime}=t_{1}-1 / \sqrt{2}$. Thus the contour $\gamma$ for $t$ is the circle centred at $1 / \sqrt{2}$ passing through $t_{1}$, and a standard calculation shows that (5.8) holds, that is,

$$
\max _{t \in \gamma}|f(S(t), t)|=\left|f\left(s_{1}, t_{1}\right)\right| .
$$

The curve $S(\gamma)$ is an oval contained in the half-disc

$$
\left\{\operatorname{Re}(s)<\frac{h}{j-k} \text { and }\left|s-\frac{h}{j-k}\right|<\frac{\sqrt{h^{2}-(j-k)^{2}}}{j-k}\right\},
$$

and is a connected component of a quartic in the $s$-plane, symmetric with respect to the real axis. A direct application of the ordinary saddle point method (that is, in $\mathbb{C}$ ) shows that for any fixed $t \in \gamma$, the modified integration contour $\delta_{t}$ for $s$, that is, by Cauchy's theorem, a suitable contour enclosing the pole $s=t$ but not the pole $s=1$ of the function

$$
\frac{s^{h}}{(1-s)^{l+k-j}(s-t)^{j+h-k}},
$$

can be chosen to be a contour passing through the saddle point $s=S(t)$ of (6.3) and satisfying (5.9). By (5.10), the constant $K_{1}$ in (4.2) is

$$
K_{1}=\log \left|f\left(s_{1}, t_{1}\right)\right|+(j-h) \log |x|+(h+j+k) \log |1-x|=13.64089760 \ldots,
$$

in accordance with [M, formula (40)]. 
For $x=-1 / \sqrt{2}$, the roots of (5.6) are

$$
\begin{aligned}
& s_{1}=-0.84282443 \ldots, \\
& s_{2}=0.71105687 \ldots+i 0.57738282 \ldots, \\
& s_{3}=\overline{s_{2}} .
\end{aligned}
$$

The corresponding values $t_{v}=T\left(s_{v}\right)$ are

$$
\begin{aligned}
& t_{1}=0.78338155 \ldots, \\
& t_{2}=-0.38306132 \ldots+i 0.70108673 \ldots, \\
& t_{3}=\overline{t_{2}},
\end{aligned}
$$

whence

$$
\begin{aligned}
& \log \left|f\left(s_{1}, t_{1}\right)\right|=-9.55296054 \ldots, \\
& \log \left|f\left(s_{2}, t_{2}\right)\right|=\log \left|f\left(s_{3}, t_{3}\right)\right|=0.14400664 \ldots
\end{aligned}
$$

According to (3.4), the integration paths in $s$ and $t$ for $I_{n}^{-}(-1 / \sqrt{2})$ are the half-lines $\left(0, \eta_{2} \infty\right)$ and $\left(0, \eta_{1} \infty\right)$ respectively, for any $\eta_{1}, \eta_{2} \in \mathbb{C}$ satisfying $\left|\eta_{1}\right|=\left|\eta_{2}\right|=1$ and $0<\arg \eta_{1}<\arg \eta_{2}<\pi$. We may rotate the integration path for $s$ to the negative half-line $(0,-\infty)$, since with this rotation the integration path does not cross the poles $s=1$ and $s=t$. Similarly, we may rotate the integration path for $t$ to $(0,+\infty)$. Therefore $I_{n}^{-}(-1 / \sqrt{2})$ is real, and by Laplace's asymptotic method we have, with the values (6.4)-(6.6),

$$
\begin{aligned}
\lim _{n \rightarrow \infty} \frac{1}{n} \log \left|I_{n}^{-}(-1 / \sqrt{2})\right| & =\log \left|f\left(s_{1}, t_{1}\right)\right|+(j-h) \log |x|+(h+j+k) \log |1-x| \\
& =-1.87753418 \ldots
\end{aligned}
$$

For $I_{n}^{+}(-1 / \sqrt{2})$, we take $s=S(t)$ on the $t$-plane deprived of $\left(-\infty, \tau_{-}\right] \cup\left[\tau_{+},+\infty\right)$ with values in $\{\operatorname{Im}(s)>0\}$. Similarly to the choice made for the modified integration paths in $I_{n}^{+}(1 / \sqrt{2})$, we define the paths $\delta$ for $s$ and $\gamma$ for $t$ by (6.1) and (6.2), but now with the value $s_{2}$ given by (6.4). It is easy to see that the curve $\gamma$ is contained in the half-plane $\operatorname{Re}(t)<0$, and, moving along $\gamma$ from zero to infinity, the point $-1 / \sqrt{2}$ lies on the left-hand side. Thus, by Cauchy's theorem, the integration paths $\delta$ and $\gamma$ are admissible for $I_{n}^{+}(-1 / \sqrt{2})$. Again as in [M], one checks that (5.8) and (5.9) hold with $\left|f\left(s_{2}, t_{2}\right)\right|$. Therefore, by $(5.10)$,

$$
\begin{aligned}
\lim _{n \rightarrow \infty} \frac{1}{n} \log \left|I_{n}^{+}(-1 / \sqrt{2})\right| & =\log \left|f\left(s_{2}, t_{2}\right)\right|+(j-h) \log |x|+(h+j+k) \log |1-x| \\
& =7.81943300 \ldots
\end{aligned}
$$

For $J_{n}(-1 / \sqrt{2})$, we take $s=S(t)$ on the $t$-plane deprived of $\left(-\infty, \tau_{-}\right] \cup\left[\tau_{+},+\infty\right)$ with values in $\{\operatorname{Im}(s)>0\}$, as before. Here we take $\varrho^{\prime}=\left|t_{2}+1 / \sqrt{2}\right|$, so that the contour $\gamma$ for $t$ is the circle centred at $-1 / \sqrt{2}$ passing through $t_{2}$ given by (6.5). The curve $S(\gamma)$ is the upper half of a quartic in the $s$-plane, and (5.8) can be easily checked with $t_{*}=t_{2}$. 
As with $J_{n}(1 / \sqrt{2})$, for any $t \in \gamma$ one can choose the contour $\delta_{t}$ for $s$ enclosing $t$ but not 1 , so that (5.9) holds. By (5.10),

$$
\begin{aligned}
\lim _{n \rightarrow \infty} \frac{1}{n} \log \left|J_{n}(-1 / \sqrt{2})\right| & =\lim _{n \rightarrow \infty} \frac{1}{n} \log \left|I_{n}^{+}(-1 / \sqrt{2})\right| \\
& =\log \left|f\left(s_{2}, t_{2}\right)\right|+(j-h) \log |x|+(h+j+k) \log |1-x| \\
& =7.81943300 \ldots
\end{aligned}
$$

Therefore the constant $K_{2}$ in (4.3) is $7.81943300 \ldots$ By (4.5),

$$
\lambda(\alpha)=0.08654193 \ldots,
$$

whence, by Theorem 4.1,

$$
\mu_{\mathbb{Q}(\sqrt{2})}(\log 2) \leq \lambda(\alpha)^{-1}=11.55509194 \ldots
$$

This improves the upper bound 12.4288 in [AV, p. 247].

(ii) $\alpha=\sqrt{2 / 3}$. Here the discussion is entirely similar to the previous case. We have $\delta(\alpha)=\Delta=2$ and $a_{0}=3$.

For $x=\alpha=\sqrt{2 / 3}$, we have the values

$$
\begin{aligned}
& s_{1}=0.96027872 \ldots, \\
& s_{2}=-0.11431453 \ldots+i 0.91498775 \ldots, \\
& s_{3}=\overline{s_{2}} \\
& t_{1}=0.87357326 \ldots, \\
& t_{2}=-0.49164065 \ldots-i 0.72210704 \ldots, \\
& t_{3}=\overline{t_{2}}, \\
& \log \left|f\left(s_{1}, t_{1}\right)\right|=40.09743371 \ldots, \\
& \log \left|f\left(s_{2}, t_{2}\right)\right|=\log \left|f\left(s_{3}, t_{3}\right)\right|=-7.95377566 \ldots
\end{aligned}
$$

For $x=-\sqrt{2 / 3}$,

$$
\begin{aligned}
s_{1} & =-0.90486575 \ldots, \\
s_{2} & =0.73660804 \ldots+i 0.59979046 \ldots, \\
s_{3} & =\overline{s_{2}} \\
t_{1} & =0.86694191 \ldots, \\
t_{2} & =-0.42028360 \ldots+i 0.76964162 \ldots, \\
t_{3} & =\overline{t_{2}}
\end{aligned}
$$




$$
\begin{aligned}
& \log \left|f\left(s_{1}, t_{1}\right)\right|=-9.89797210 \ldots, \\
& \log \left|f\left(s_{2}, t_{2}\right)\right|=\log \left|f\left(s_{3}, t_{3}\right)\right|=-0.14931027 \ldots
\end{aligned}
$$

We get

$$
\mu_{\mathbb{Q}(\sqrt{3 / 2})}(\log (3 / 2)) \leq 10.80627515 \ldots \quad([\mathrm{AV}]: 12.1383) .
$$

(iii) Here we take $\alpha$ to be the root of the polynomial $5 x^{3}-5 x^{2}+1$ satisfying $\operatorname{Im}(\alpha)>0$, whence

$$
\alpha=0.69030472 \ldots+i 0.22125188 \ldots
$$

We have $\delta(\alpha)=3 / 2, \Delta=3, a_{0}=5$.

For $x=\alpha$ we have the values

$$
\begin{aligned}
s_{1}= & 0.93578505 \ldots+i 0.05801374 \ldots, \\
s_{2}= & -0.21584264 \ldots+i 0.84783681 \ldots, \\
s_{3}= & -0.00091193 \ldots-i 0.88372537 \ldots, \\
t_{1}= & 0.78980202 \ldots+i 0.16573657 \ldots, \\
t_{2}= & -0.30680221 \ldots-i 0.74487075 \ldots, \\
t_{3}= & -0.58219187 \ldots+i 0.56069651 \ldots, \\
& \log \left|f\left(s_{1}, t_{1}\right)\right|=28.54154439 \ldots, \\
& \log \left|f\left(s_{2}, t_{2}\right)\right|=-8.05635599 \ldots, \\
& \log \left|f\left(s_{3}, t_{3}\right)\right|=-7.23841357 \ldots
\end{aligned}
$$

For $I_{n}^{+}(\alpha)$, the modified integration paths for $s$ and $t$ are the half-line $\delta=\mathbb{R}^{+} s_{2}$ and the curve $\gamma=T\left(\mathbb{R}^{+} s_{2}\right)$, respectively. For $I_{n}^{-}(\alpha)$, we choose $\delta=\mathbb{R}^{+} s_{3}$ and $\gamma=T\left(\mathbb{R}^{+} s_{3}\right)$, respectively. For $J_{n}(\alpha)$, we choose the integration contour $\gamma$ for $t$ to be the circle centred at $\alpha$ passing through $t_{1}$, and for any $t \in \gamma$ we choose the modified contour $\delta_{t}$ for $s$ according to the ordinary saddle point method, that is, passing through the saddle point $s=S(t) \in C_{3}$.

For $x=\bar{\alpha}$, we plainly have

$$
I_{n}^{+}(\bar{\alpha})=\overline{I_{n}^{-}(\alpha)}, \quad I_{n}^{-}(\bar{\alpha})=\overline{I_{n}^{+}(\alpha)}, \quad J_{n}(\bar{\alpha})=\overline{J_{n}(\alpha)} .
$$

For $x=\beta=-0.38060945 \ldots$, the real root of $5 x^{3}-5 x^{2}+1$, we have the values

$$
\begin{aligned}
& s_{1}=-0.61911354 \ldots, \\
& s_{2}=0.61552629 \ldots+i 0.48568772 \ldots, \\
& s_{3}=\overline{s_{2}}
\end{aligned}
$$




$$
\begin{aligned}
t_{1} & =0.50571018 \ldots, \\
t_{2} & =-0.25782970 \ldots+i 0.46901975 \ldots, \\
t_{3} & =\overline{t_{2}}, \\
\log \left|f\left(s_{1}, t_{1}\right)\right| & =-8.15371480 \ldots, \\
\log \left|f\left(s_{2}, t_{2}\right)\right| & =\log \left|f\left(s_{3}, t_{3}\right)\right|=1.03644597 \ldots
\end{aligned}
$$

Here the choices of the modified integration paths for $I_{n}^{+}(\beta), I_{n}^{-}(\beta)$ and $J_{n}(\beta)$ are quite similar to the choices made in example (i) above for $x=-1 / \sqrt{2}$. Thus $I_{n}^{-}(\beta)$ is real, and by Laplace's asymptotic method

$$
\lim _{n \rightarrow \infty} \frac{1}{n} \log \left|I_{n}^{-}(\beta)\right|=\log \left|f\left(s_{1}, t_{1}\right)\right|+(j-h) \log |\beta|+(h+j+k) \log (1-\beta) .
$$

For $I_{n}^{+}(\beta)$, the modified integration paths for $s$ and $t$ are the half-line $\delta=\mathbb{R}^{+} s_{2}$ and $\gamma=T\left(\mathbb{R}^{+} s_{2}\right)$, respectively. For $J_{n}(\beta)$ we choose the integration contour $\gamma$ for $t$ to be the circle centred at $\beta$ passing through $t_{2}$, and for any $t \in \gamma$ we choose the contour $\delta_{t}$ for $s$ passing through the saddle point $s=S(t)$ in the half-plane $\{\operatorname{Im}(s)>0\}$.

We obtain

$$
\mu_{\mathbb{Q}(\alpha)}(\log \alpha) \leq 6.93729219 \ldots \quad([\mathrm{AV}]: 7.105) .
$$

(iv) $\alpha=e^{i \pi / 6}=\frac{1}{2}(\sqrt{3}+i)$. This is an algebraic integer of degree four, since its minimal polynomial in $\mathbb{Z}[x]$ is $x^{4}-x^{2}+1$. Hence $\delta(\alpha)=2, \Delta=4, a_{0}=1$.

The algebraic conjugates are $\alpha_{1}=\alpha=e^{i \pi / 6}, \alpha_{2}=e^{-i \pi / 6}, \alpha_{3}=e^{i 5 \pi / 6}, \alpha_{4}=e^{-i 5 \pi / 6}$. If $x$ is any one of these numbers, then

$$
\left|s_{1}\right|=\left|s_{2}\right|=\left|s_{3}\right|=\left|t_{1}\right|=\left|t_{2}\right|=\left|t_{3}\right|=1,
$$

an easy consequence of (5.7).

For $x=\alpha=e^{i \pi / 6}$,

$$
\begin{aligned}
s_{1}= & 0.99449517 \ldots+i 0.10478236 \ldots, \\
s_{2}= & -0.33491983 \ldots+i 0.94224662 \ldots, \\
s_{3}= & 0.07702719 \ldots-i 0.99702899 \ldots, \\
t_{1}= & 0.93974079 \ldots+i 0.34188776 \ldots, \\
t_{2}= & -0.24351342 \ldots-i 0.96989752 \ldots, \\
t_{3}= & -0.81006282 \ldots+i 0.58634309 \ldots, \\
& \log \left|f\left(s_{1}, t_{1}\right)\right|=25.38692084 \ldots, \\
& \log \left|f\left(s_{2}, t_{2}\right)\right|=-9.07190278 \ldots, \\
& \log \left|f\left(s_{3}, t_{3}\right)\right|=-7.68078113 \ldots
\end{aligned}
$$


For $I_{n}^{+}(\alpha)$, we choose the integration paths $\delta=\mathbb{R}^{+} s_{2}$ for $s$ and $\gamma=T\left(\mathbb{R}^{+} s_{2}\right)$ for $t$. For $I_{n}^{-}(\alpha)$, we choose $\delta=\mathbb{R}^{+} s_{3}$ and $\gamma=T\left(\mathbb{R}^{+} s_{3}\right)$. For $J_{n}(\alpha)$, we choose the integration contour $\gamma$ for $t$ to be the circle centred at $\alpha$ passing through $t_{1}$, and for any $t \in \gamma$ we choose the contour $\delta_{t}$ for $s$ passing through the saddle point $s=S(t) \in C_{3}$.

For $x=\alpha_{2}=\bar{\alpha}$,

$$
I_{n}^{+}(\bar{\alpha})=\overline{I_{n}^{-}(\alpha)}, \quad I_{n}^{-}(\bar{\alpha})=\overline{I_{n}^{+}(\alpha)}, \quad J_{n}(\bar{\alpha})=\overline{J_{n}(\alpha)} .
$$

For $x=\alpha_{3}=e^{i 5 \pi / 6}$,

$$
\begin{aligned}
s_{1}= & 0.84948835 \ldots+i 0.52760737 \ldots, \\
s_{2}= & -0.96700551 \ldots+i 0.25475542 \ldots, \\
s_{3}= & 0.68091462 \ldots-i 0.73236279 \ldots, \\
t_{1}= & -0.15888485 \ldots+i 0.98729712 \ldots, \\
t_{2}= & 0.93287114 \ldots-i 0.36021025 \ldots, \\
t_{3}= & -0.74348417 \ldots-i 0.66875352 \ldots, \\
& \log \left|f\left(s_{1}, t_{1}\right)\right|=1.84282546 \ldots, \\
& \log \left|f\left(s_{2}, t_{2}\right)\right|=-10.34643759 \ldots, \\
& \log \left|f\left(s_{3}, t_{3}\right)\right|=-2.61651939 \ldots,
\end{aligned}
$$

For $I_{n}^{+}\left(\alpha_{3}\right)$, we choose the integration paths $\delta=\mathbb{R}^{+} s_{2}$ and $\gamma=T\left(\mathbb{R}^{+} s_{2}\right)$. For $I_{n}^{-}\left(\alpha_{3}\right)$, we choose $\delta=\mathbb{R}^{+} s_{3}$ and $\gamma=T\left(\mathbb{R}^{+} s_{3}\right)$. For $J_{n}\left(\alpha_{3}\right)$, we choose $\gamma$ to be the circle centred at $\alpha_{3}$ passing through $t_{1}$, and for any $t \in \gamma$ we choose the contour $\delta_{t}$ passing through $s=S(t)$ in $\{\operatorname{Im}(s)>0\}$.

For $x=\alpha_{4}=\overline{\alpha_{3}}$,

$$
I_{n}^{+}\left(\overline{\alpha_{3}}\right)=\overline{I_{n}^{-}\left(\alpha_{3}\right)}, \quad I_{n}^{-}\left(\overline{\alpha_{3}}\right)=\overline{I_{n}^{+}\left(\alpha_{3}\right)}, \quad J_{n}\left(\overline{\alpha_{3}}\right)=\overline{J_{n}\left(\alpha_{3}\right)} .
$$

We find

$$
\mu_{\mathbb{Q}(i, \sqrt{3})}(i \pi / 6)=\mu_{\mathbb{Q}(\sqrt{3})}(\pi) \leq 36.17593210 \ldots \quad([\mathrm{AV}]: 46.9075) .
$$

6.2. We give some applications of Theorem 4.2 with $\alpha=\sqrt{a /(a+1)}$, for the integers $a$ between 6 and 15 . Thus we obtain nonquadraticity measures of $\log (1+1 / a)$ over the field $\mathbb{Q}(\sqrt{1+1 / a})$.

Here we choose the exponents in the function $f(s, t)$ depending on the integer $a$ and satisfying both (5.3) and $2 h=j+k$, so that the nonquadraticity measure obtained for each $a$ depends only on the rational parameter $1 / 2<h / j<1$. The applications of the $\mathbb{C}^{2}$-saddle point method to $I_{n}^{ \pm}(\sqrt{a /(a+1)}), J_{n}(\sqrt{a /(a+1)}), I_{n}^{ \pm}(-\sqrt{a /(a+1)})$, $J_{n}(-\sqrt{a /(a+1)})$ are entirely similar to the case of $1 / \sqrt{2}$ for Theorem 4.1 .

We get the results in Table 1. 
TABLE 1.

\begin{tabular}{rrrcc}
\hline$a$ & \multicolumn{1}{c}{$h$} & \multicolumn{1}{c}{$j$} & $h / j$ & $\mu_{2, \mathrm{Q}(\sqrt{1+1 / a})}(\log (1+1 / a))<$ \\
\hline 6 & 11 & 12 & $0.916666 \ldots$ & $877.596309 \ldots$ \\
7 & 181 & 195 & $0.928205 \ldots$ & $178.047043 \ldots$ \\
8 & 13 & 14 & $0.928571 \ldots$ & $106.287388 \ldots$ \\
9 & 92 & 99 & $0.929292 \ldots$ & $79.296423 \ldots$ \\
10 & 165 & 177 & $0.932203 \ldots$ & $65.055453 \ldots$ \\
11 & 97 & 104 & $0.932692 \ldots$ & $56.198271 \ldots$ \\
12 & 71 & 76 & $0.934210 \ldots$ & $50.142909 \ldots$ \\
13 & 29 & 31 & $0.935483 \ldots$ & $45.725407 \ldots$ \\
14 & 31 & 33 & $0.939393 \ldots$ & $42.340246 \ldots$ \\
15 & 79 & 84 & $0.940476 \ldots$ & $39.656780 \ldots$ \\
\hline
\end{tabular}

\section{Acknowledgement}

We are indebted to the referee for helpful comments and suggestions on an earlier version of this paper.

\section{References}

[AV] F. Amoroso and C. Viola, 'Approximation measures for logarithms of algebraic numbers', Ann. Sc. Norm. Super Pisa (4) 30 (2001), 225-249.

[H1] M. Hata, 'Rational approximations to $\pi$ and some other numbers', Acta Arith. 63 (1993), 335-349.

[H2] M. Hata, ' $\mathbb{C}^{2}$-saddle method and Beukers' integral', Trans. Amer. Math. Soc. 352 (2000), $4557-4583$.

[M] R. Marcovecchio, 'The Rhin-Viola method for log 2', Acta Arith. 139 (2009), 147-184.

[RV1] G. Rhin and C. Viola, 'On a permutation group related to $\zeta(2)$ ', Acta Arith. 77 (1996), 23-56.

[RV2] G. Rhin and C. Viola, 'The group structure for $\zeta(3)$ ', Acta Arith. 97 (2001), 269-293.

[RV3] G. Rhin and C. Viola, 'The permutation group method for the dilogarithm', Ann. Sc. Norm. Super Pisa (5) 4 (2005), 389-437.

[S] W. M. Schmidt, Diophantine Approximation, Lecture Notes in Mathematics, 785 (Springer, 1980).

[V] C. Viola, 'Hypergeometric functions and irrationality measures', in: Analytic Number Theory (Kyoto, 1996), London Mathematical Society Lecture Note Series, 247 (Cambridge University Press, Cambridge, 1997), pp. 353-360.

RAFFAELE MARCOVECCHIO, Fakultät für Mathematik, Universität Wien, Nordbergstraße 15, 1090 Wien, Austria e-mail: marcovr8@univie.ac.at

CARLO VIOLA, Dipartimento di Matematica, Università di Pisa, Largo B. Pontecorvo 5, 56127 Pisa, Italy

e-mail: viola@dm.unipi.it 